\title{
Safety and immunogenicity of the SARS-CoV-2 BNT162b1 mRNA vaccine in younger and older Chinese adults: a randomized, placebo-controlled, double-blind phase 1 study
}

\author{
Jingxin $\mathrm{Li}^{1,10}$, Aimin Hui ${ }^{2,10 凶}$, Xiang Zhang ${ }^{3}$, Yumei Yang ${ }^{4}$, Rong Tang ${ }^{1}$, Huayue Ye ${ }^{5,6}$, Ruiru Ji², \\ Mei Lin7, Zhongkui Zhu ${ }^{3}$, Özlem Türeci ${ }^{8}$, Eleni Lagkadinou ${ }^{8}$, Siyue Jia ${ }^{1}$, Hongxing Pan ${ }^{1}$, Fuzhong Peng ${ }^{5,7}$, \\ Zhilong $\mathrm{Ma}^{3}$, Zhenggang Wu${ }^{7}$, Xiling Guo', Yunfeng Shi', Alexander Muik ${ }^{8}{ }^{8}$, Uğur Șahin ${ }^{8}$, Li Zhu $\mathbb{1}^{7 凶}$ \\ and Fengcai Zhu ${ }^{1,9 凶}$
}

\begin{abstract}
An effective vaccine is needed to end the severe acute respiratory syndrome coronavirus 2 (SARS-CoV-2) pandemic. Here, we assess the preliminary safety, tolerability and immunogenicity data from an ongoing single-center (in Jiangsu province, China), parallel-group, double-blind phase 1 trial of the vaccine candidate BNT162b1 in 144 healthy SARS-CoV-2-naive Chinese participants. These participants are randomized 1:1:1 to receive prime and boost vaccinations of $10 \mu \mathrm{g}$ or $30 \mu \mathrm{g}$ BNT162b1 or placebo, given $21 \mathrm{~d}$ apart, with equal allocation of younger (aged 18-55 years) and older adults (aged 65-85 years) to each treatment group (ChiCTR2000034825). BNT162b1 encodes the SARS-CoV-2 spike glycoprotein receptor-binding domain (RBD) and is one of several messenger RNA-based vaccine candidates under clinical investigation. Local reactions and systemic events were generally dose dependent, transient and mild to moderate. Fever was the only grade 3 adverse event. BNT162b1 induced robust interferon- $\gamma$ T cell responses to a peptide pool including the RBD in both younger and older Chinese adults, and geometric mean neutralizing titers reached 2.1-fold (for younger participants) and 1.3-fold (for the older participants) that of a panel of COVID19 convalescent human sera obtained at least $14 \mathrm{~d}$ after positive SARS-CoV-2 polymerase chain reaction test. In summary, BNT162b1 has an acceptable safety profile and produces high levels of humoral and T cell responses in an Asian population.
\end{abstract}

S ince the identification of the first cases of coronavirus disease 2019 (COVID-19) caused by severe acute respiratory syndrome coronavirus 2 (SARS-CoV-2) in December 2019, the resulting pandemic has led to more than 130 million confirmed COVID-19 cases, and more than 2.8 million deaths worldwide as of 6 April 2021 (refs. ${ }^{1-3}$ ). The development of an efficacious COVID-19 vaccine is currently the world's leading research priority ${ }^{4}$. According to a survey conducted by the World Health Organization, 87 vaccine candidates are being assessed in clinical trials, of which 23 are ongoing phase 3 trials, and an additional 186 vaccine candidates are in preclinical trials ${ }^{5}$.

Compared with other traditional approaches such as those involving inactivated or live virus vaccines or recombinant proteins, the messenger RNA-based prophylactic vaccine platform is a recently developed vaccine technology ${ }^{6-8}$. The RNA-based platform has enabled rapid vaccine development in response to the COVID-19 pandemic and provides flexibility in antigen $\operatorname{design}^{9,10}$. The candidate vaccine BNT162b1 is one of two lipid nanoparticle (LNP) formulated, pharmacologically optimized ${ }^{11,12}$, 1-methylpseudouridine nucleoside-modified mRNA (modRNA) ${ }^{13}$ vaccines against SARS-CoV-2, developed in Project Lightspeed, which was launched by BioNTech in collaboration with Pfizer and
Fosun Pharma. BNT162b1 encodes a trimerized, secreted version of the SARS-CoV-2 spike (S) glycoprotein receptor-binding domain $(\mathrm{RBD})^{14}$, in which the RBD is a key target of virus-neutralizing antibodies $^{15,16}$. The BioNTech-Pfizer-Fosun RNA vaccine development program involves a series of clinical trials that are currently being conducted in Germany, the United States and China ${ }^{17-19}$, among other countries. At the time of writing, BNT162b2, a second vaccine candidate from the same platform, which encodes a prefusion stabilized, full-length SARS-CoV-2 S protein, has been selected as a pivotal candidate based on the totality of data obtained in the US and German phase 1 and 2 trials and in non-human primate challenge studies $^{14,17,19,20}$. BNT162b2 has been authorized or approved for emergency or temporary use or granted conditional marketing authorization in more than 50 countries worldwide, including the European Union countries, the United States and the United Kingdom ${ }^{21,22}$.

The safety, tolerability and immunogenicity of the BNT162b1 vaccine have been reported recently from the ongoing phase 1,2 and 3 NCT04368728 trials in the United States ${ }^{17,18}$, and from the phase 1 and 2 NCT04380701 and EudraCT 2020-001038-36 vaccine trials in Germany ${ }^{19}$.

Here, we present a clinical report on an mRNA-based vaccine in a Chinese population from the China-based ChiCTR2000034825-

${ }^{1} \mathrm{NHC}$ Key Laboratory of Enteric Pathogenic Microbiology, Jiangsu Provincial Center for Disease Control and Prevention, Nanjing, China. ${ }^{2}$ Fosun Pharma, Boston, MA, USA. ${ }^{3}$ Taizhou City Center for Disease Control and Prevention, Taizhou, China. ${ }^{4}$ Fosun Pharma, Beijing, China. ${ }^{5}$ Taizhou Vaccine Clinical Research Center, Taizhou, China. ${ }^{6}$ Department of Pharmacy, Army Medical University, Chongqing, China. ${ }^{7}$ Taizhou People's Hospital, Taizhou, China. ${ }^{8}$ BioNTech SE, Mainz, Germany. ${ }^{9}$ Center for Global Health, Nanjing Medical University, Nanjing, China. ${ }^{10}$ These authors contributed equally: Jingxin Li, Aimin Hui.凶e-mail: aimin.hui@fosunpharma.com; tzheart@126.com; jszfc@vip.sina.com 


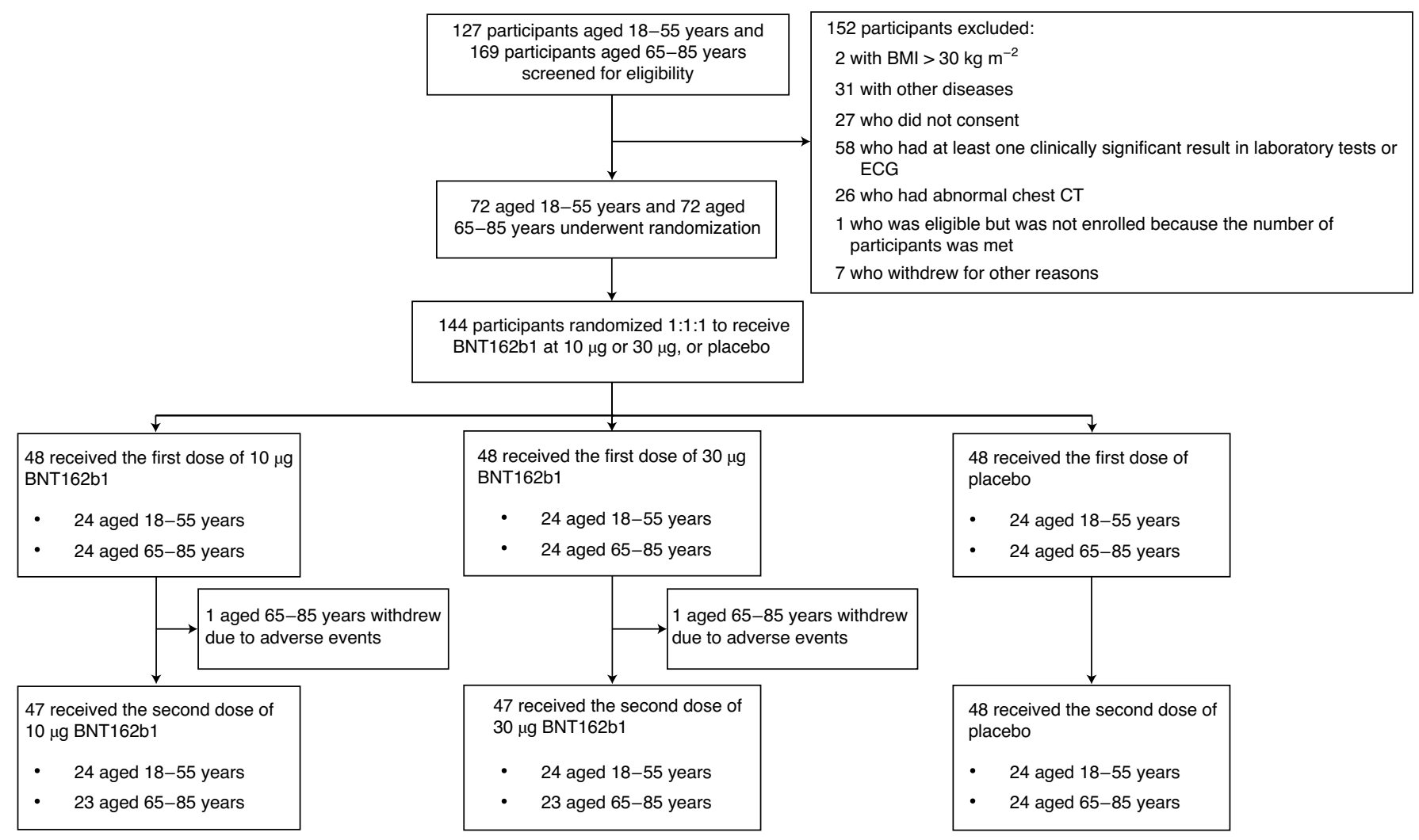

Fig. 1 | Study flow diagram. BMI, body mass index; CT, computed tomography; ECG, electrocardiograph.

NCT04523571 study investigating the preliminary safety and immunogenicity data of the BNT162b1 vaccine in healthy, young and older Chinese participants.

\section{Results}

Study design and analysis set. Between 18 July 2020 and 14 August 2020, a total of 296 adults aged between 18 and 55 years or between 65 and 85 years were screened at Taizhou Vaccine Clinical Research Center in Jiangsu Province, China. A total of 144 eligible participants consented to participate in the trial and were randomized 1:1:1 to receive prime and boost doses of BNT162b1 at $10 \mu \mathrm{g}$ or $30 \mu \mathrm{g}$, or two placebo (saline) doses, $21 \mathrm{~d}$ apart (Fig. 1). Participants in each age group were allocated equally to each treatment group (48 participants per dose or placebo group, consisting of 24 participants aged 18-55years and 24 participants aged $65-85$ years). Following the priming dose, two participants in the $65-85$ years age group (one in the $10 \mu \mathrm{g}$ group and one in the $30 \mu \mathrm{g}$ group) withdrew before receiving the boost dose (Supplementary Table 1). The demographic characteristics of the participants are listed in Table 1. In the three treatment groups the mean age of the younger participants ranged from 37.9 to 42.0 years, and the mean age of the older participants ranged from 68.5 to 70.7 years, with equal gender distribution across treatment groups. People with a known allergy, hypersensitivity or intolerance to vaccines or to any excipients in the tested BNT162b1 mRNA vaccine were excluded from the study. The medical history or other existing underlying disorders of the participants were similar across treatment groups, except for hypertension, which was noted at a higher prevalence in all of the older participant groups (i.e., including the placebo group) in the BNT162b1 older participant groups at baseline.

Preliminary safety and tolerability data. Adverse events were graded according to the guidelines for vaccine clinical trials issued by the US Food and Drug Administration (FDA) ${ }^{23}$ (Table 2) and the National Medical Products Administration (NMPA), China ${ }^{24}$ (Table 3). In the $7 \mathrm{~d}$ after the prime or boost vaccination, 21 (88\%) of the younger participants in the $10 \mu \mathrm{g}$ BNT162b1 dose group and $24(100 \%)$ of the younger participants in the $30 \mu \mathrm{g}$ BNT162b1 dose group reported at least one solicited adverse reaction, versus four (17\%) of the younger participants in the placebo group. A similar trend was seen for older participants, in that $83 \%$ of participants (20 of 24 ) in the $10 \mu \mathrm{g}$ BNT162b1 dose group and $92 \%$ of participants ( 22 of 24 ) in the $30 \mu \mathrm{g}$ BNT162b1 dose group reported at least one solicited adverse reaction, versus $8 \%$ of participants ( 2 of 24 ) in the placebo group (Table 2). Reactogenicity was dose-level dependent and was most evident in the group receiving $30 \mu \mathrm{g}$ BNT162b1. The most common solicited adverse reactions reported were injection site pain, fever, headache, fatigue, malaise, joint pain, muscle pain and chills (Tables 2 and 3). The adverse events were transient and were either managed with simple standard of care or resolved spontaneously. Most of the reported adverse reactions were mild or moderate in severity, and resolved in the first $7 \mathrm{~d}$ after each BNT162b1 dose for the prime and boost vaccinations. None of the injection site reactions was graded as severe (grade 3). Grade 3 fever was the only severe systemic adverse reaction associated with BNT162b1 vaccination, and it was predominantly observed in participants who received $30 \mu \mathrm{g}$ BNT162b1. Grade 3 fever was reported in $17 \%$ of younger participants ( 4 of 24 ) and in $8 \%$ of older participants ( 2 of 24 ) who received $30 \mu \mathrm{g}$ BNT162b1, and in $4 \%$ of younger participants (1 of 24) who received $10 \mu \mathrm{g}$ BNT162b1 (Table 2 and Supplementary Table 2). One male participant in the older age group experienced an episodic grade 3 fever accompanied by pain and pruritus at the injection site after the prime BNT162b1 dose at $30 \mu \mathrm{g}$, and electively withdrew before receiving the boost vaccination. Mild or moderate headache was predominantly observed in the younger participants who received $30 \mu \mathrm{g}$ BNT162b1 (79\%, 19 
Table 1 | Baseline characteristics of the participants by age group

\begin{tabular}{|c|c|c|c|c|c|c|}
\hline \multirow{2}{*}{ Characteristic } & \multicolumn{3}{|c|}{ Younger participants ( $18-55$ years) } & \multicolumn{3}{|c|}{ Older participants (65-85 years) } \\
\hline & $10 \mu \mathrm{g}$ BNT162b1 & $30 \mu g$ BNT162b1 & Placebo & $10 \mu \mathrm{g}$ BNT162b1 & $30 \mu \mathrm{g}$ BNT162b1 & Placebo \\
\hline No. of participants & 24 & 24 & 24 & 24 & 24 & 24 \\
\hline \multicolumn{7}{|l|}{ Sex, $n(\%)$} \\
\hline Male & $12(50)$ & $12(50)$ & $12(50)$ & $12(50)$ & $12(50)$ & $12(50)$ \\
\hline Female & $12(50)$ & $12(50)$ & $12(50)$ & $12(50)$ & $12(50)$ & $12(50)$ \\
\hline \multicolumn{7}{|l|}{ Race, $n(\%)$} \\
\hline Chinese (Asian) & $24(100)$ & $24(100)$ & $24(100)$ & $24(100)$ & $24(100)$ & $24(100)$ \\
\hline Other & 0 & 0 & 0 & 0 & 0 & 0 \\
\hline \multicolumn{7}{|c|}{ Medical history or existing disorder, $n(\%)$} \\
\hline Cardiac ischemia & $2(8)$ & $2(8)$ & $2(8)$ & 0 & 0 & 0 \\
\hline Blood uric acid increased & $2(8)$ & $1(4)$ & $1(4)$ & 0 & 0 & 0 \\
\hline Hypertension & $3(13)$ & 0 & $1(4)$ & $12(50)$ & $9(38)$ & $7(29)$ \\
\hline Diabetes & 0 & 0 & 0 & $1(4)$ & $2(8)$ & $1(4)$ \\
\hline Gastric inflammation & 0 & 0 & 0 & 0 & 0 & $2(8)$ \\
\hline Others $^{a}$ & $3(13)$ & $5(21)$ & $1(4)$ & $3(13)$ & $3(13)$ & $4(17)$ \\
\hline
\end{tabular}

aTonsillitis, Helicobacter infection, human papilloma virus infection, periodontitis, electrocardiogram high voltage, lymphadenopathy, anemia, hepatic cyst, oropharyngeal discomfort, hyperthyroidism, non-infective gingivitis, hyperlipidemia, benign prostatic hyperplasia, prostatitis, unilateral blindness, cerebral infarct, limb injury, deformity of spine, urinary calculus and lymphadenopathy.

of 24 participants); far fewer younger participants who received placebo $(13 \%, 3$ of 24$)$ and older participants who received $30 \mu \mathrm{g}$ BNT162b1 $(8 \%, 2$ of 24$)$ had mild or moderate headache (Tables 2 and 3).

No pre-specified trial-halting criteria were met during the study. Only one serious adverse event was reported by an older participant, which was considered as not related to the vaccine or study procedure (they were involved in an accident that prevented them from receiving the boost dose). The overall frequencies of the post-vaccination local adverse reactions were similar after the BNT162b1 prime and boost doses. Some systemic adverse reactions such as fever (oral temperature $>38^{\circ} \mathrm{C}$ ), headache, fatigue and malaise occurred more commonly after the BNT162b1 boost dose than after the prime dose in younger adults (Extended Data Fig. 1). Fever generally resolved in the $48 \mathrm{~h}$ after onset. In contrast to the younger participants, older participants did not have increased reactogenicity after the BNT162b1 boost dose (Extended Data Fig. 2).

There were no changes reported in participant blood pressure or respiratory rate across the different treatment groups after BNT162b1 administration. Transient increases in temperature and pulse rate in the $24 \mathrm{~h}$ after the prime and the boost vaccinations were noted in both younger and older participants, especially in the $30 \mu \mathrm{g}$ BNT162b1 dose group (Extended Data Fig. 3). The most common abnormalities in laboratory values from baseline were transient decreases in lymphocyte and platelet counts and increases in C-reactive protein (CRP) level. All of the laboratory abnormalities were self-limited and resolved in a short period of time without clinical symptoms (Extended Data Fig. 4). These data are in line with the results seen in the EudraCT 2020-001038-36 study and in other mRNA vaccine trials that have found CRP level ${ }^{25,26}$ and lymphocyte count ${ }^{27}$ to act as pharmacodynamic markers for the mode of action of these vaccines.
Results of a long-term follow up of safety and tolerability up to 12 months following vaccination for participants in the BNT162b1 vaccination dose cohorts will be published separately.

Vaccine-induced antibody responses. SARS-CoV-2-neutralization titers as well as RBD-binding and S1-binding antibody titers were assessed at baseline (on $\mathrm{d} 1$, immediately before the priming dose on that day), 7 and $21 \mathrm{~d}$ after the priming dose ( $\mathrm{d} 8$ and 22 , respectively), and 7 and $21 \mathrm{~d}$ after the boost dose (d 29 and 43 , respectively). The SARS-CoV-2-neutralizing titer is defined as the reciprocal of the highest sample dilution that protects at least $50 \%$ of the cells from cytopathic effects (CPEs). The BNT162b1-induced neutralizing antibody responses in vaccinated participants were simultaneously tested against a panel of human COVID-19 convalescent sera obtained at least $14 \mathrm{~d}$ after polymerase chain reaction (PCR)-confirmed diagnosis from 24 Asian patients hospitalized with COVID-19, 21 (87.5\%) of whom had symptomatic disease. All of the participants were seronegative at baseline (on $\mathrm{d} 1$, immediately before the priming dose on that day) and had a modest vaccine-induced antibody response $21 \mathrm{~d}$ after the priming dose (d22), which further increased on d 29 (Fig. 2 and Supplementary Table 3). The highest neutralizing titers were observed on $\mathrm{d} 43$ (that is, $21 \mathrm{~d}$ after the BNT162b1 boost dose) for both the younger and older adults in the BNT162b1 group, indicating a continuous increase in neutralizing titers in this group of Asian participants after $\mathrm{d} 29$. On $\mathrm{d} 43$ the $50 \%$ neutralizing geometric mean titer (GMT) in the $10 \mu \mathrm{g}$ and the $30 \mu \mathrm{g}$ dose groups was 232.9 (95\% confidence interval (CI), 151.3 to 358.5 ) and 254.0 (95\% CI, 184.6 to 349.4 ) in the younger participants, and 80.0 (95\% CI, 49.2 to 130.2$)$ and 160.0 (95\% CI, 96.7 to 264.6$)$ in the older participants, respectively, which was significantly higher than that on $\mathrm{d} 29$ for both the dose groups and the age groups (all $P<0.0001$ ). The peak SARS-CoV-2-neutralizing antibody GMT for the younger participants in the $10 \mu \mathrm{g}$ and $30 \mu \mathrm{g}$ 
Table 2 | Solicited adverse reactions in the $7 \mathrm{~d}$ after prime or boost vaccinations, and unsolicited adverse reactions until $\mathrm{d} 50$, graded by FDA criteria, by age group

\begin{tabular}{|c|c|c|c|c|c|c|c|c|}
\hline \multirow[b]{3}{*}{ Adverse reactions } & \multicolumn{4}{|c|}{ Younger participants ( $18-55$ years) } & \multicolumn{4}{|c|}{ Older participants (65-85 years) } \\
\hline & \multicolumn{4}{|c|}{ BNT162b1 } & \multicolumn{4}{|c|}{ BNT162b1 } \\
\hline & $\begin{array}{l}10 \mu \mathrm{g} \\
(n=24)\end{array}$ & $\begin{array}{l}30 \mu \mathrm{g} \\
(n=24)\end{array}$ & $\begin{array}{l}\text { Placebo } \\
(n=24)\end{array}$ & $P$ value & $\begin{array}{l}10 \mu \mathrm{g} \\
(n=24)\end{array}$ & $\begin{array}{l}30 \mu g \\
(n=24)\end{array}$ & $\begin{array}{l}\text { Placebo } \\
(n=24)\end{array}$ & $P$ value \\
\hline \multicolumn{9}{|c|}{ Solicited adverse reactions within $7 \mathrm{~d}, n(\%)$} \\
\hline Any & $21(88)$ & $24(100)$ & $4(17)$ & $<0.0001$ & $20(83)$ & $22(92)$ & $2(8)$ & $<0.0001$ \\
\hline Grade 3 & $1(4)$ & $4(17)$ & 0 & 0.0015 & 0 & $2(8)$ & 0 & 0.3239 \\
\hline Any & $21(88)$ & $24(100)$ & $2(8)$ & $<0.0001$ & $18(75)$ & $21(88)$ & 0 & $<0.0001$ \\
\hline Grade 3 & 0 & 0 & 0 & - & 0 & 0 & 0 & - \\
\hline Pain & $21(88)$ & $23(96)$ & $2(8)$ & $<0.0001$ & $16(67)$ & $21(88)$ & 0 & $<0.0001$ \\
\hline Redness & $6(25)$ & $8(33)$ & 0 & 0.0059 & $3(13)$ & $4(17)$ & 0 & 0.1492 \\
\hline Swelling & $5(21)$ & $7(29)$ & 0 & 0.0137 & 0 & $5(21)$ & 0 & 0.0091 \\
\hline Grade 3 & $1(4)$ & $4(17)$ & 0 & 0.0015 & 0 & $2(8)$ & 0 & 0.3239 \\
\hline Fever $^{a}$ & $9(38)$ & $18(75)$ & 0 & $<0.0001$ & $1(4)$ & $16(67)$ & 0 & $<0.0001$ \\
\hline Grade $3^{b}$ & $1(4)$ & $4(17)$ & 0 & 0.1185 & 0 & $2(8)$ & 0 & 0.3239 \\
\hline Headache & $11(46)$ & $19(79)$ & $3(13)$ & $<0.0001$ & $1(4)$ & $2(8)$ & 0 & 0.7682 \\
\hline Fatigue & $12(50)$ & $16(67)$ & 0 & $<0.0001$ & $3(13)$ & $8(33)$ & 0 & 0.0045 \\
\hline Malaise & $8(33)$ & $9(38)$ & 0 & 0.0013 & $2(8)$ & $4(17)$ & $1(4)$ & 0.4858 \\
\hline Joint pain & $4(17)$ & $10(42)$ & $1(4)$ & 0.0067 & 0 & $1(4)$ & 0 & 1.0000 \\
\hline Muscle pain & $2(8)$ & $10(42)$ & 0 & $<0.0001$ & 0 & $1(4)$ & 0 & 1.0000 \\
\hline Chills & $4(17)$ & 7 (29) & 0 & 0.0118 & $1(4)$ & $4(17)$ & 0 & 0.1185 \\
\hline Fever $^{c}$ & 0 & 0 & 0 & - & 0 & $1(4)$ & 0 & 1.0000 \\
\hline Temperature intolerance & $2(8)$ & $6(25)$ & 0 & 0.0230 & 0 & $4(17)$ & 0 & 0.0310 \\
\hline Injection site discomfort & $3(13)$ & $4(17)$ & 0 & 0.1492 & $2(8)$ & $3(13)$ & 0 & 0.3580 \\
\hline Injection site pruritus & $2(8)$ & $3(13)$ & 0 & 0.3580 & 0 & $1(4)$ & 0 & 1.0000 \\
\hline Pain not at injection site & $1(4)$ & $1(4)$ & 0 & 1.0000 & 0 & 0 & 0 & - \\
\hline Dizziness & $3(13)$ & $1(4)$ & 0 & 0.3142 & 0 & $3(13)$ & 0 & 0.1018 \\
\hline Blood uric acid increased & $1(4)$ & $1(4)$ & 0 & 1.0000 & $2(8)$ & $1(4)$ & $2(8)$ & 1.0000 \\
\hline
\end{tabular}

${ }^{a}$ Oral temperature of $\geq 38.0^{\circ} \mathrm{C}$ (ref. ${ }^{23}$ ). ${ }^{b}$ Oral temperature of $39-40^{\circ} \mathrm{C}\left(\right.$ ref. ${ }^{23}$ ). ${ }^{c}$ One participant experienced grade 3 fever accompanied by pain, itching and pruritus at the injection site after the prime dose, and electively withdrew before the boost vaccination. Grade 3 reactions are defined as vaccination-related events that are severe enough to prevent normal activities. A participant was counted only once in a specific reaction category, regardless of the number of episodes of the adverse reaction. Only unsolicited adverse reactions reported by two or more participants are listed.

dose groups was 1.9-fold and 2.1-fold the GMT of the entire convalescent serum panel $(n=24, \mathrm{GMT}=119.9,95 \% \mathrm{CI}, 70.4$ to 203.9$)$ (Supplementary Table 4). The d 43 GMT of younger participants vaccinated with $30 \mu \mathrm{g}$ BNT162b1 was significantly higher than the GMT of the entire convalescent serum panel $(P=0.042)$. In the older participants, the peak neutralizing antibody GMTs on $\mathrm{d} 43$ in the $10 \mu \mathrm{g}$ and $30 \mu \mathrm{g}$ dose groups were 0.7 -fold and 1.3 -fold the GMT of the entire convalescent serum panel, respectively $(P=0.532$ for the $30 \mu \mathrm{g}$ dose group). Participants who received the $30 \mu \mathrm{g}$ dose appeared to have a relatively higher virus-neutralizing antibody response than those who received the $10 \mu \mathrm{g}$ dose. The older participants generally had a lower peak neutralizing antibody response than the younger participants.

For all of the younger participants, seroconversion (a fourfold or greater increase in neutralizing antibody GMT above baseline) had occurred by $\mathrm{d} 43$, while the rate of seroconversion in the older recipients was $91 \%$ at the $10 \mu \mathrm{g}$ dose and $96 \%$ at the $30 \mu \mathrm{g}$ dose (Extended Data Fig. 5). A post-hoc multivariate repeated measurements analysis of neutralizing antibody GMT on d 1, 22, 29 and 43 across the treatment and age groups showed that GMT significantly 
Table 3 | Solicited adverse reactions in the $14 \mathrm{~d}$ after prime or boost vaccinations, and unsolicited adverse reactions until $\mathrm{d} 50$, graded by NMPA criteria, by age group

\begin{tabular}{|c|c|c|c|c|c|c|c|c|}
\hline \multirow[b]{3}{*}{ Adverse reactions } & \multicolumn{4}{|c|}{ Younger participants ( $18-55$ years) } & \multicolumn{4}{|c|}{ Older participants (65-85 years) } \\
\hline & \multicolumn{4}{|c|}{ BNT162b1 } & \multicolumn{4}{|c|}{ BNT162b1 } \\
\hline & $\begin{array}{l}10 \mu \mathrm{g} \\
(n=24)\end{array}$ & $\begin{array}{l}30 \mu \mathrm{g} \\
(n=24)\end{array}$ & $\begin{array}{l}\text { Placebo } \\
(n=24)\end{array}$ & $P$ value & $\begin{array}{l}10 \mu \mathrm{g} \\
(n=24)\end{array}$ & $\begin{array}{l}30 \mu g \\
(n=24)\end{array}$ & $\begin{array}{l}\text { Placebo } \\
(n=24)\end{array}$ & $P$ value \\
\hline \multicolumn{9}{|c|}{ Solicited adverse reactions within $14 \mathrm{~d}, n(\%)$} \\
\hline & $21(88)$ & $24(100)$ & $4(17)$ & $<0.0001$ & $21(88)$ & $23(96)$ & $2(8)$ & $<0.0001$ \\
\hline Grade 3 & $3(13)$ & $9(38)$ & 0 & 0.0015 & 0 & $2(8)$ & 0 & 0.3239 \\
\hline Any & $21(88)$ & $24(100)$ & $2(8)$ & $<0.0001$ & $18(75)$ & $21(88)$ & 0 & $<0.0001$ \\
\hline Grade 3 & 0 & 0 & 0 & - & 0 & 0 & 0 & - \\
\hline Pain & $21(88)$ & $23(96)$ & $2(8)$ & $<0.0001$ & $16(67)$ & $21(88)$ & 0 & $<0.0001$ \\
\hline Redness & $6(25)$ & $8(33)$ & 0 & 0.0059 & $3(13)$ & $4(17)$ & 0 & 0.1492 \\
\hline Swelling & $5(21)$ & 7 (29) & 0 & 0.0137 & 0 & $5(21)$ & 0 & 0.0091 \\
\hline Grade 3 & $3(13)$ & $9(38)$ & 0 & 0.0015 & 0 & $2(8)$ & 0 & 0.3239 \\
\hline Fever $^{a}$ & $14(58)$ & $21(88)$ & $1(4)$ & $<0.0001$ & $7(29)$ & 19 (79) & $1(4)$ & $<0.0001$ \\
\hline Grade $3^{b}$ & $3(13)$ & $9(38)$ & 0 & 0.0015 & 0 & $2(8)$ & 0 & 0.3239 \\
\hline Headache & $11(46)$ & $19(79)$ & $3(13)$ & $<0.0001$ & $1(4)$ & $2(8)$ & 0 & 0.7682 \\
\hline Fatigue & $12(50)$ & $16(67)$ & 0 & $<0.0001$ & $3(13)$ & $8(33)$ & 0 & 0.0045 \\
\hline Malaise & $8(33)$ & $9(38)$ & 0 & 0.0013 & $2(8)$ & $4(17)$ & $1(4)$ & 0.4858 \\
\hline Joint pain & $4(17)$ & $10(42)$ & $1(4)$ & 0.0067 & 0 & $1(4)$ & 0 & 1.0000 \\
\hline Muscle pain & $2(8)$ & $10(42)$ & 0 & $<0.0001$ & 0 & $1(4)$ & 0 & 1.0000 \\
\hline Chills & $4(17)$ & 7 (29) & 0 & 0.0118 & $1(4)$ & $4(17)$ & 0 & 0.1185 \\
\hline Fever & 0 & 0 & 0 & - & 0 & $1(4)$ & 0 & 1.0000 \\
\hline Temperature intolerance & $2(8)$ & $6(25)$ & 0 & 0.0230 & 0 & $4(17)$ & 0 & - \\
\hline Injection site discomfort & $3(13)$ & $4(17)$ & 0 & 0.1492 & $2(8)$ & $3(13)$ & 0 & 0.3580 \\
\hline Injection site pruritus & $2(8)$ & $3(13)$ & 0 & 0.3580 & 0 & $1(4)$ & 0 & 1.0000 \\
\hline Pain not at injection site & $1(4)$ & $1(4)$ & 0 & 1.0000 & 0 & 0 & 0 & - \\
\hline Dizziness & $3(13)$ & $1(4)$ & 0 & 0.3142 & 0 & $3(13)$ & 0 & 0.1018 \\
\hline Blood uric acid increased & $1(4)$ & $1(4)$ & 0 & 1.0000 & $2(8)$ & $1(4)$ & $2(8)$ & 1.0000 \\
\hline
\end{tabular}

${ }^{a}$ Axillary temperature of $\geq 37.3^{\circ} \mathrm{C}\left(\right.$ ref. ${ }^{24}$ ). ${ }^{b}$ Axillary temperature of 38.5 to $<39.5^{\circ} \mathrm{C}\left(\right.$ ref. ${ }^{24}$ ). ${ }^{c}$ One participant experienced grade 3 fever accompanied by pain, itching and pruritus at the injection site after the prime dose, and electively withdrew before the boost vaccination. Grade 3 reactions are defined as vaccination-related events that are severe enough to prevent normal activities. A participant was counted only once in a specific reaction category, regardless of the number of episodes of the adverse reaction. Only unsolicited adverse reactions reported by two or more participants are listed.

correlated with treatment (BNT162b1 vaccine versus placebo), time after treatment (days after immunization), participant age and combinations thereof (time $\times$ treatment interaction, age $\times$ treatment interaction and time $\times$ age interaction) (Supplementary Table 5).

In line with the neutralizing antibody titers, BNT162b1 induced high levels of S1-binding and RBD-binding immunoglobulin- $\gamma$ (IgG) GMTs after the prime-boost regimen. Binding IgG GMTs from the younger participants who received $30 \mu \mathrm{g}$ BNT162b1 reached the upper limit of quantification in the ELISA test by d 29 ( $7 \mathrm{~d}$ after the boost dose), indicating that the analysis of the
S1-binding and RBD-binding IgG titers in this participant subset could be compromised. Putting aside this methodological limitation, the S1-binding IgG levels were positively correlated with the neutralizing antibody titers across all of the time points evaluated in the participants, regardless of age group or dose level, with a correlation coefficient of 0.66 for younger participants and 0.55 for older participants (both $P<0.0001$ ) (Extended Data Fig. 6a,b).

Vaccine-induced T cell responses. Vaccine-induced Tcell responses were analyzed on $\mathrm{d} 29$ ( $7 \mathrm{~d}$ after the boost dose) and on 
$\mathrm{d} 43$ ( $21 \mathrm{~d}$ after the boost dose), using a direct ex vivo interferon- $\gamma$ (IFN- $\gamma$ ) enzyme-linked immunosorbent spot (ELISpot) assay with bulk peripheral blood mononuclear cells (PBMCs) (Fig. 3). T cell responses detected for the vaccinated dose groups were compared with those for the placebo control. PBMCs were stimulated overnight with either of two overlapping peptide pools representing the amino-terminal SARS-CoV-2 S protein portion (amino acids 1-643, S pool 1 (Sp1), which includes the RBD sequence (amino acids 319-541)) and the carboxy-terminal S protein portion (amino acids 633-1273, S pool 2 (Sp2)), or a combination of human leukocyte antigen (HLA) class I-restricted cytomegalovirus (CMV), Epstein-Barr virus (EBV) and influenza virus T cell epitopes (CEF peptide pool) as a positive control.

Seven days after the BNT162b1 boost dose (d29), a significant increase in Sp1-reactive T cells compared with the placebo control was detectable independent of dose and age group (Fig. 3a,b, all $P<0.0001$ ). Younger participants (that is, those aged $18-55$ years) who had received $10 \mu \mathrm{g}$ or $30 \mu \mathrm{g}$ BNT162b1 had geometric mean IFN- $\gamma^{+}$spot counts of 36 per $10^{5}$ PBMCs (95\% CI, 21.44 to 60.42 ) and 50.45 per $10^{5}$ PBMCs (95\% CI, 29.63 to 85.87 ), respectively. In the older participants (that is, those aged 65-85 years), somewhat lower geometric mean IFN- $\gamma^{+}$spot counts of 18.9 per $10^{5}$ PBMCs (95\% CI, 3.67 to 97.29 ) in the $10 \mu \mathrm{g}$ dose group and 24.96 per $10^{5}$ PBMCs (95\% CI, 5.96 to 104.6) in the $30 \mu \mathrm{g}$ dose group were noted. The younger and older adult placebo controls had a geometric mean IFN- $\gamma^{+}$spot count of 1.89 per $10^{5}$ PBMCs (95\% CI, 1.30 to 2.74 ) and 1.92 per $10^{5}$ PBMCs (95\% CI, 0.58 to 6.35 ), respectively. In the younger participants receiving the prime-boost BNT162b1 regimen, Sp1-reactive T cells at $\mathrm{d} 43$ ( $21 \mathrm{~d}$ after the boost dose) were slightly decreased compared with d 29 (geometric mean IFN- $\gamma^{+}$spot counts of 30.99 per $10^{5}$ PBMCs (95\% CI, 14.79 to 65.34 ) and 49.52 per $10^{5}$ PBMCs (95\% CI, 31.72 to 77.31 ), respectively); no blood samples were collected at $\mathrm{d} 43$ from the older participants. In line with BNT162b1 encoding the SARS-CoV-2 S RBD antigen, and as would be expected given that the Sp2 peptide pool does not cover the RBD of the spike protein encoded by the vaccine, no significant change in Sp2-reactive Tcell number compared with the placebo control was noted.

In aggregate, BNT162b1 vaccine-induced Sp1-reactive bulk T cell responses were higher (in younger adults) or similar (in older adults) to individual memory responses against the CEF peptide pool positive control (Fig. 3c). For younger adults, the magnitude of Sp1-specific T cell responses correlated positively with SARS-CoV2-neutralizing antibody GMTs (Extended Data Fig. 6c, $P=0.0004$ ), indicating a convergent development of the humoral and cellular adaptive immunity. No statistically significant correlation was observed for the older adults (Extended Data Fig. 6d, $P=0.0723$ ).

\section{Discussion}

Both BNT162b1 and BNT162b2 were granted Fast Track designation by the US FDA in July 2020. This trial was conducted in China in parallel with other trials testing BNT162 vaccine candidates in the United States and Germany ${ }^{17-20}$. BNT162b1 and BNT162b2 have been shown to elicit similar dose-dependent SARS-CoV2-neutralizing GMTs, at levels similar to or in excess of those in the convalescent COVID-19 serum panels used as comparators ${ }^{17}$. However, BNT162b2 has been shown to have a milder systemic reactogenicity profile. Moreover, the $\mathrm{BNT} 162 \mathrm{~b} 2$ vaccine induces T cell responses against the RBD, S1 and S2 regions of the S protein, indicating immune recognition of multiple, independent MHC I and II epitopes, while as shown here and elsewhere ${ }^{17}$, BNT162b1 induces a response against only the $\mathrm{S} 1$ region.

Given the favorable reactogenicity and immunogenicity profile of BNT162b2, it was selected as the global lead candidate in late July 2020. However, few data are available on the safety and immunogenicity of mRNA vaccines in Asian populations. This preliminary report of the clinical trial of the SARS-CoV-2 vaccine candidate BNT162b1 provides an evaluation of both the safety and the immunogenicity profile of such an mRNA vaccine in a Chinese population. Although further development of BNT162b1 in China is not foreseen, this report will contribute to understanding the profile of mRNA vaccines in Chinese populations and will serve to inform ongoing development of similar products to tackle the pandemic.

BNT162b1 has been shown to exhibit a broadly similar immunogenicity profile to that of BNT162b2 (modRNA encoding the full-length SARS-CoV-2 spike glycoprotein, derived from the same nucleoside-modified platform), and to induce strong SARS-CoV-2 S-binding and SARS-CoV-2-neutralizing antibody responses and strong Tcell responses in younger and older healthy adults in Germany and the United States ${ }^{17-20}$. In line with these findings, our data show that prime-boost vaccination with $10 \mu \mathrm{g}$ and $30 \mu \mathrm{g}$ BNT162b1 vaccine is safe and induces a strong humoral and cellular immune response in younger and older healthy Asian adults (18-55 years and 65-85 years of age).

In agreement with the US and German trial results ${ }^{18,19}$, pain at the injection site was the most common solicited adverse reaction reported in the Chinese participants in this trial. No injection site reactions were graded as severe (grade 3). Also in line with prior reports, fever, headache, fatigue, malaise, joint pain, muscle pain and chills were confirmed in this study as the most common systemic solicited adverse reactions. As previously reported, these adverse events were transient and were either managed with simple standard of care or resolved spontaneously. The observed reactogenicity to BNT162b1 was dose dependent, and a higher frequency of adverse events was generally observed after the second dose. Mild or moderate headache was more prevalent in the younger Chinese participants who received $30 \mu \mathrm{g}$ BNT162b1 (79\%, 19 of 24) than in younger Chinese participants who received placebo (13\%, 3 of 24$)$ or older Chinese participants who received $30 \mu \mathrm{g}$ BNT162b1 (8\%, 2 of 24). A mild safety profile was observed in older Chinese people vaccinated with BNT162b1, with fever being the only severe adverse reaction observed (in 2 of 24 participants, 8\%). Older Chinese participants who received BNT162b1 at a dose of $30 \mu \mathrm{g}$ also had a lower frequency of several systemic adverse reactogenicity events than those previously reported in non-Asian older populations (any grade of headache (8\% versus $75 \%$ ), fatigue (33\% versus $67 \%$ ), chills (17\% versus $33 \%)$, joint pain (4\% versus $16.7 \%)$ ), indicating a potential favorable systemic reactogenicity profile in the Asian older population compared with non-Asian populations ${ }^{17}$, although it should be noted that differences may be attributable to the relatively

Fig. 2 | BNT162b1-induced 50\% virus-neutralizing antibody titers and specific RBD-binding and S1-binding antibody responses, compared with placebo.

a-c, Serum samples were obtained at baseline (before prime, d1), d 8 and 22 (before boost), and d 29 and 43 (after boost) in the younger adult group, and at baseline (before prime, d1), d 22 (before boost), and d 29 and 43 (after boost) in the older adult group. A panel of human COVID-19 convalescent sera $(n=24)$ was obtained at least $14 \mathrm{~d}$ after PCR-confirmed diagnosis in patients with COVID-19. Fifty percent SARS-CoV-2-neutralizing antibody GMTs (a), RBD-binding (b) and S1-binding (c) antibody GMTs measured by ELISA are shown. Each point represents a serum sample, and each bar represents the GMT with $95 \% \mathrm{Cl}$. The dashed line indicates the lower limit of quantification (LLOQ). The LLOQ was 1:10 for the virus-neutralizing antibody test and 1:100 for ELISA. Comparison of antibody titers between time points in the same group was performed using the Wilcoxon matched-pairs signed-rank test; peak neutralizing antibody responses of participants in each dose group were compared to the convalescent sera using the Mann-Whitney U-test. NS, not significant; ${ }^{\star} P<0.05 ;{ }^{\star \star \star \star} P<0.0001$. 
small sample size evaluated in this phase 1 study. Furthermore, laboratory investigations showed transient decreases in lymphocyte counts predominantly in the younger recipients who received BNT162b1 at a dose of $30 \mu \mathrm{g}$, in line with the reported mode of action of the vaccine, which causes the redistribution of lymphocytes into lymphoid tissues by innate immune stimulation ${ }^{27}$. In summary, the preliminary data complement and extend the reporting of BNT162b1 and other RNA-based vaccine candidates from clinical trials conducted in Germany and the United States to an Asian population ${ }^{16,17,19,20}$.
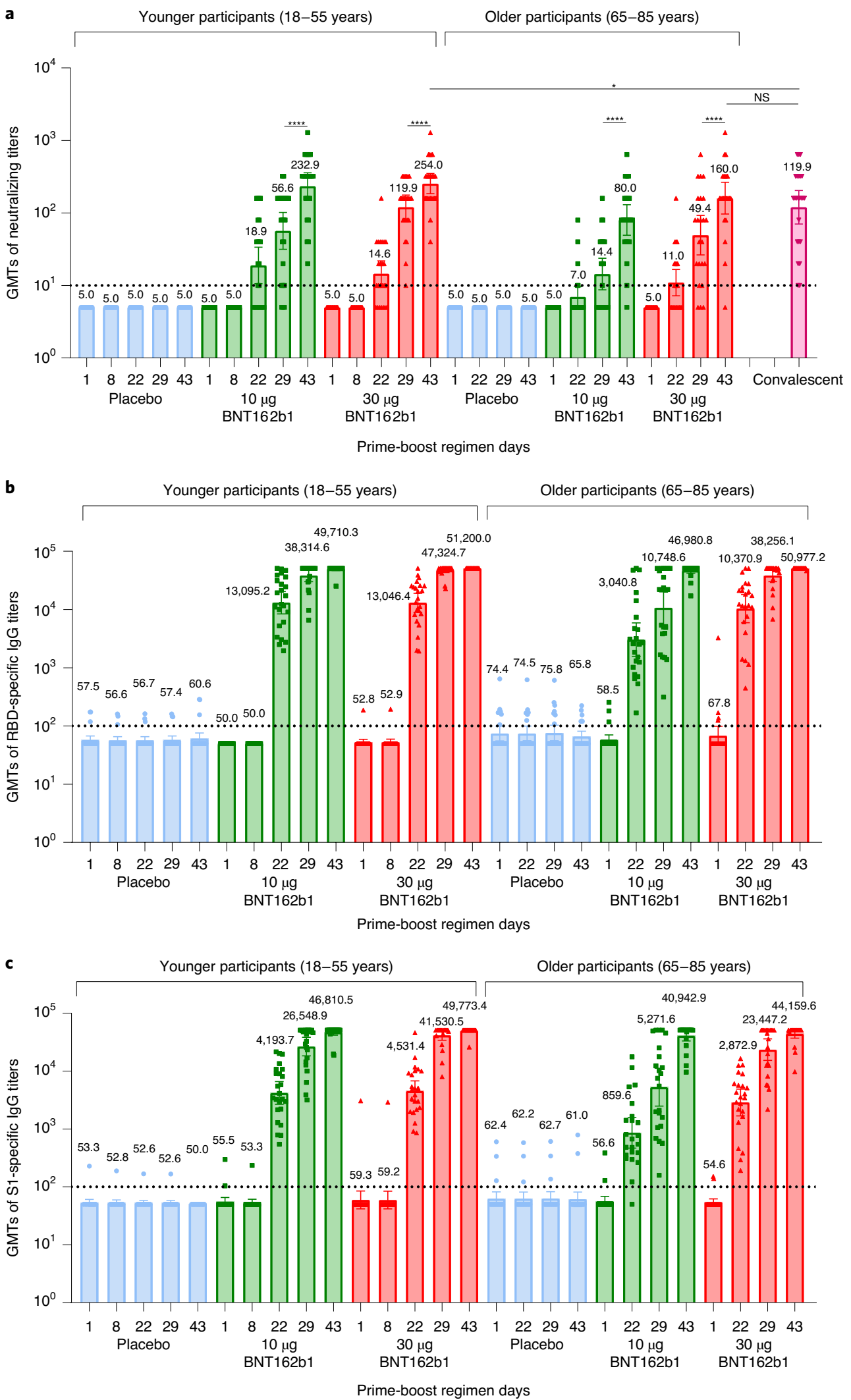
a

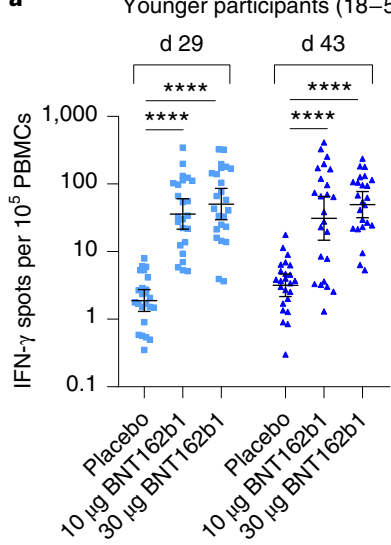

Sp1

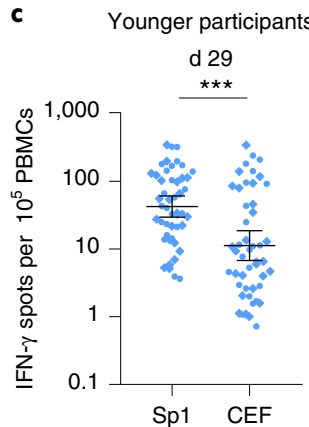

b

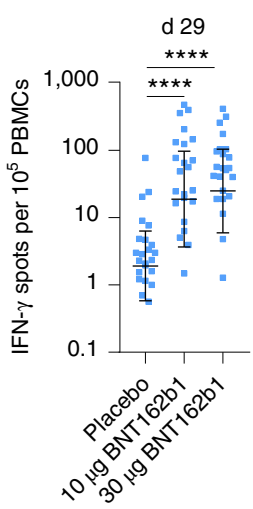

Sp1 d 29

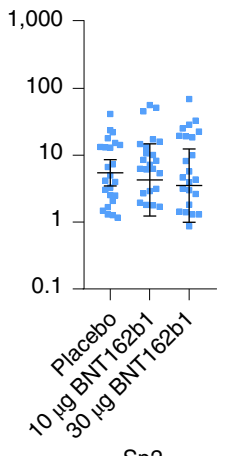

Older participants d 29

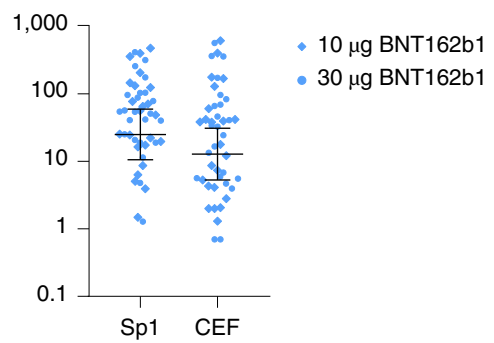

Fig. 3 | BNT162b1-induced T cell responses in vaccinated participants compared with placebo. Bulk PBMCs obtained on d 29 and 43 (7 d and $21 \mathrm{~d}$ after boost) were stimulated overnight with either of two overlapping peptide pools representing different portions of the wild-type sequence of SARS-CoV-2 S (N-terminal Sp1 and the C-terminal Sp2), for assessment in direct ex vivo IFN- $\gamma$ ELISpot assay, or the CEF peptide pool (immune-dominant HLA class I T cell epitopes of CMV, EBV and influenza virus) as a positive control. Each dot represents the mean spot count from triplicate wells for one participant, after subtraction of the medium-only control. a,b, SARS-CoV-2 specific T cell response in younger adult participants aged $18-55$ years (a) and older adult participants aged 65-85 years (b). c, Comparison of Sp1-reactive T cell responses and individual memory responses against CEF on d 29. Group geometric mean spot counts and $95 \% \mathrm{Cls}$ are shown. Mann-Whitney $U$-test was used to determine significant differences. ${ }^{\star \star \star} P<0.001 ;{ }^{\star \star \star \star} P<0.0001$.

Prime-boost immunization with the vaccine candidate BNT162b1 was effective at eliciting specific humoral and cellular immune responses, with a clear boost effect of the second vaccination on antibody titers in both younger and older adults. Two doses of $30 \mu \mathrm{g}$ BNT162 b1, $21 \mathrm{~d}$ apart, induced a strong immune response in terms of virus-neutralizing antibody to SARS-CoV-2, which in younger patients was significantly higher than, and in older adults was similar to, that observed in a panel of convalescent serum samples from Asian people with COVID-19. The highest neutralizing antibody titers were observed on $\mathrm{d} 43$ (that is, $21 \mathrm{~d}$ after the BNT162b1 boost dose) regardless of age in this study. In the previously reported German trial of BNT162b1 (NCT04380701), participants had a higher level of neutralizing titers at d 29 compared with that at $\mathrm{d} 43$, indicating that the peak titers occurred earlier ${ }^{19}$. In the US trial of BNT162b1 (NCT04368728), the highest level of neutralizing titers was observed at $\mathrm{d} 35$, indicating a continuous increase in neutralizing titer level in the participants after $\mathrm{d} 29$ (ref. ${ }^{17}$ ). Here, we also noted a continuous increase in neutralizing titer level in the participants receiving BNT162b1 after d 29. However, given that the neutralizing titers at $\mathrm{d} 43$ were not measured in the US trial, and that the neutralizing titers at $\mathrm{d} 35$ were not measured in the present study, we cannot tell whether the peak of the neutralizing responses in Chinese adults is similar to or later than that in the participants in the United States. Nevertheless, although the timing of the peak neutralizing antibody response induced by BNT162b1 may vary slightly between different populations or in different trials, peak antibody titers are most likely to occur $1-3$ weeks after the boost vaccination, based on the collective findings. To definitively address this question, the temporal pattern of BNT162b1-induced humoral immunogenicity needs to be studied in Asian and non-Asian populations side by side, ideally by one laboratory.

Given that the vaccine candidate BNT162b1 encodes a trimerized, secreted version of the SARS-CoV-2 S RBD, the vaccine recipients in this study demonstrated a significant $\mathrm{T}$ cell response specific to peptides in the $\mathrm{N}$ terminus of the SARS-CoV-2 S protein (including the RBD region; pool $\mathrm{Sp} 1$ ), but not to peptides in the C-terminal S protein portion (pool Sp2). At d 29, BNT162b1-vaccinated younger and older participants had significantly stronger Sp1-specific T cell responses compared with participants receiving placebo. The observed cellular immune response was more widely variable in the older participants than in the younger participants. The vaccine candidate BNT162b2, which encodes an optimized prefusion stabilized full-length $S$ protein, showed a broader immune response spectrum, inducing strong $T$ cell responses against both the Sp1 and Sp2 peptide pools ${ }^{14}$. Nonetheless, the data show that the BNT162b1 vaccine candidate at a dose of $30 \mu \mathrm{g}$ is highly immunogenic and is capable of eliciting strong humoral and cell-mediated responses in healthy Chinese adults.

This study has several limitations, including data interpretation based on a limited, small sample size, and the restriction to only adult Chinese participants of 18 years of age and older in this study. Future evaluation of the tolerability, safety and immunogenicity of SARS-COV-2 prophylactic RNA vaccines in Asian children and adolescent populations is warranted. Next, although 
the serum-neutralizing responses elicited by the BNT162b1 vaccine candidate were similar to those of human convalescent serum panels, the level of serological immunity needed to protect against COVID-19 has not yet been determined ${ }^{28}$. In addition, the human convalescent serum panels that have been used in different trials are not standardized between laboratories, and each has a different distribution of patient characteristics and time points of collection, which imposes challenges in the comparison of immunogenicity across studies and different vaccines. Furthermore, the persistence of BNT162b1-elicited immune responses is not yet known. However, assessment of vaccine-induced immune persistence is planned in the study protocol (Supplementary Data 1) and these data will be collected and reported later.

In summary, this study confirms the tolerability and favorable immunogenicity profile of the RNA-based SARS-CoV-2 vaccine candidate BNT162b1, and extends the reporting of BNT162b1 and other RNA-based vaccine candidates from clinical trials conducted in Germany and the United States to an Asian population. BNT162b1 encodes a relatively small RBD immunogen, which might induce a narrower spectrum of neutralizing antibodies that are less robust to potential antigenic drift of SARS-CoV-2 compared with BNT162b2, which encodes a full-length spike immunogen. Pfizer and BioNTech have recently announced that the vaccine candidate BNT162b2 was found to be more than 95\% effective in preventing COVID-19 in a phase 3 pivotal study, with equally strong efficacy observed in participants under and over 65 years of age (NCT04368728) ${ }^{29}$. Currently, a phase 2 clinical trial of the vaccine candidate BNT162b2 is underway in 960 Chinese adults between 18 and 85 years of age in China, consistent with BioNTech's global partnering strategy for the development of the SARS-CoV-2 vaccine (ChiCTR2000040044 and NCT04523571).

\section{Online content}

Any methods, additional references, Nature Research reporting summaries, source data, extended data, supplementary information, acknowledgements, peer review information; details of author contributions and competing interests; and statements of data and code availability are available at https://doi.org/10.1038/ s41591-021-01330-9.

Received: 28 December 2020; Accepted: 23 March 2021; Published online: 22 April 2021

\section{References}

1. Gudbjartsson, D. F. et al. Spread of SARS-CoV-2 in the Icelandic population. N. Engl. J. Med. 382, 2302-2315 (2020).

2. Huang, C. et al. Clinical features of patients infected with 2019 novel coronavirus in Wuhan, China. Lancet 395, 497-506 (2020).

3. World Health Organization. Weekly epidemiological update on COVID-19-6 April 2021. https://www.who.int/publications/m/item/ weekly-epidemiological-update-on-covid-19---6-april-2021 (2021).

4. Jackson, L. A. et al. An mRNA vaccine against SARS-CoV-2: preliminary report. N. Engl. J. Med. 383, 1920-1931 (2020).

5. World Health Organization. Draft landscape and tracker of COVID-19 candidate vaccines. https://www.who.int/publications/m/item/draftlandscape-of-covid-19-candidate-vaccines (2021).

6. Pardi, N. et al. Nucleoside-modified mRNA vaccines induce potent $\mathrm{T}$ follicular helper and germinal center B cell responses. J. Exp. Med. 215, 1571-1588 (2018).
7. Pardi, N. et al. Nucleoside-modified mRNA immunization elicits influenza virus hemagglutinin stalk-specific antibodies. Nat. Commun. 9, 3361 (2018).

8. Sahin, U., Kariko, K. \& Türeci, O. mRNA-based therapeutics: developing a new class of drugs. Nat. Rev. Drug Discov. 13, 759-780 (2014).

9. Corbett, K. S. et al. SARS-CoV-2 mRNA vaccine design enabled by prototype pathogen preparedness. Nature 586, 567-571 (2020).

10. Anderson, E. J. et al. Safety and immunogenicity of SARS-CoV-2 mRNA1273 vaccine in older adults. N. Engl. J. Med. 383, 2427-2438 (2020)

11. Holtkamp, S. et al. Modification of antigen-encoding RNA increases stability, translational efficacy, and T-cell stimulatory capacity of dendritic cells. Blood 108, 4009-4017 (2006).

12. Orlandini von Niessen, A. G. et al. Improving mRNA-based therapeutic gene delivery by expression-augmenting $3^{\prime}$ UTRs identified by cellular library screening. Mol. Ther. 27, 824-836 (2019).

13. Karikó, K. et al. Incorporation of pseudouridine into mRNA yields superior nonimmunogenic vector with increased translational capacity and biological stability. Mol. Ther. 16, 1833-1840 (2008).

14. Vogel, A. B. et al. BNT162b vaccines protect rhesus macaques from SARS-CoV-2. Nature https://doi.org/10.1038/s41586-021-03275-y (2021).

15. Wrapp, D. et al. Cryo-EM structure of the 2019-nCoV spike in the prefusion conformation. Science 367, 1260-1263 (2020).

16. Brouwer, P. J. M. et al. Potent neutralizing antibodies from COVID-19 patients define multiple targets of vulnerability. Science 369, 643-650 (2020).

17. Walsh, E. E. et al. Safety and immunogenicity of two RNA-based Covid-19 vaccine candidates. N. Engl. J. Med. 383, 2439-2450 (2020).

18. Mulligan, M. J. et al. Phase I/II study of COVID-19 RNA vaccine BNT162b1 in adults. Nature 586, 589-593 (2020).

19. Sahin, U. et al. COVID-19 vaccine BNT162b1 elicits human antibody and $\mathrm{T}_{\mathrm{H}} 1 \mathrm{~T}$ cell responses. Nature 586, 594-599 (2020).

20. Sahin, U. et al. BNT162b2 induces SARS-CoV-2-neutralising antibodies and T cells in humans. Preprint at medRxiv https://doi.org/10.1101/2020. 12.09.20245175 (2020)

21. US Food and Drug Administration. Pfizer-BioNTech COVID-19 Vaccine. https://www.fda.gov/emergency-preparedness-and-response/coronavirusdisease-2019-covid-19/pfizer-biontech-covid-19-vaccine\#additional

22. Medicines \& Healthcare Products Regulatory Agency. Summary of the Public Assessment Report for Pfizer/BioNTech COVID-19 Vaccine. https://www.gov uk/government/publications/regulatory-approval-of-pfizer-biontech-vaccinefor-covid-19/summary-public-assessment-report-for-pfizerbiontechcovid-19-vaccine

23. US Food and Drug Administration. Toxicity Grading Scale for Healthy Adult and Adolescent Volunteers Enrolled in Preventive Vaccine Clinical Trials. https://www.fda.gov/regulatory-information/search-fda-guidance-documents/ toxicity-grading-scale-healthy-adult-and-adolescent-volunteers-enrolledpreventive-vaccine-clinical (2007).

24. National Medical Products Administration. Guidelines for Adverse Event Classification Standards for Clinical Trials of Preventive Vaccines. https:// www.nmpa.gov.cn/xxgk/ggtg/qtggtg/20191231111901460.html (2019).

25. Tsai, M. Y. et al. Effect of influenza vaccine on markers of inflammation and lipid profile. J. Lab. Clin. Med. 145, 323-327 (2005).

26. Doener, F. et al. RNA-based adjuvant CV8102 enhances the immunogenicity of a licensed rabies vaccine in a first-in-human trial. Vaccine 37, 1819-1826 (2019).

27. Kamphuis, E., Junt, T., Waibler, Z., Forster, R. \& Kalinke, U. Type I interferons directly regulate lymphocyte recirculation and cause transient blood lymphopenia. Blood 108, 3253-3261 (2006).

28. Hodgson, S. H. et al. What defines an efficacious COVID-19 vaccine? A review of the challenges assessing the clinical efficacy of vaccines against SARS-CoV-2.Lancet Infect. Dis. 21, e26-e35 (2021).

29. Business Wire. Pfizer and BioNTech Announce Vaccine Candidate Against COVID-19 Achieved Success in First Interim Analysis from Phase 3 Study. https://www.businesswire.com/news/home/20201109005539/en (9 November 2020).

Publisher's note Springer Nature remains neutral with regard to jurisdictional claims in published maps and institutional affiliations.

(C) The Author(s), under exclusive licence to Springer Nature America, Inc. 2021 


\section{Methods}

Study design and participants. We performed a randomized, placebo-controlled, double-blind, parallel-group phase 1 trial in healthy young adults between 18 and 55 years of age, and older adults between 65 and 85 years of age, in Taizhou, Jiangsu Province, China. Participants were in overall good health as established by medical history, physical examination, and laboratory tests at the screening visit. Both male and female participants were included and agreed to use contraception during the trial. Participants who were pregnant or breastfeeding were excluded. Participants who tested positive for SARS-CoV-2 via a commercial rapid diagnostic kit for IgM or IgG antibody to SARS-CoV-2 (Livzon diagnostics) or via testing with a pharyngeal swab nucleic acid diagnostic test (Fosun Pharma) were excluded. The presence of features of COVID-19 on chest computed tomography was a further exclusion criterion. Participants with serious cardiovascular disease or chronic conditions such as uncontrolled diabetes and hypertension, AIDS, hepatitis B and hepatitis $\mathrm{C}$ were excluded. A complete list of the inclusion and exclusion criteria is provided in Supplementary Table 6. Written informed consent was obtained from each participant before the start of the study.

The study was conducted in accordance with the Declaration of Helsinki and Good Clinical Practice. The trial protocol (Supplementary Data 1) was reviewed and approved by the Chinese NMPA and the institutional review board of the Jiangsu Provincial Center of Disease Control and Prevention.

This trial was registered with the Chinese Clinical Trial Registry (ChiCTR2000034825) and with ClinicalTrials.gov (NCT04523571). The firstpatient-in date was 28 July 2020. Enrollment was completed on 14 August 2020.

Randomization and blinding. Eligible participants between 18 and 55 years of age were enrolled in the younger age group, and participants between 65 years and 85 years of age were enrolled in the older age group. Participants were randomized at a ratio of 1:1:1 to receive low-dose BNT162b1 or high-dose BNT162b1 or placebo. Participants were stratified by gender, using a web-based interactive response technology (IRT) system. The blocked randomization list was generated by an independent statistician using SAS version 9.4. This exploratory phase 1 study was not powered to detect statistically significant differences, and a sufficient sample size was selected to facilitate the evaluation of the safety of the investigational vaccine.

Authorized unblinded pharmacists prepared the vaccines or placebo according to the allocation of participants through the IRT system, and nurses gave the investigational products to participants. The unblinded staff had no further involvement in the trial, and were forbidden to disclose allocation information to others. All other investigators, participants, laboratory staff and the sponsor remained blinded throughout the trial.

Vaccine, placebo and vaccination. BNT162b1 consists of a Good Manufacturing Practice-grade mRNA drug substance encoding the SARS-CoV-2 spike glycoprotein RBD antigen, formulated with lipids to obtain the RNA-LNP drug product. Vaccine was transported and supplied as a buffered-liquid solution for intramuscular injection, and stored at $-80^{\circ} \mathrm{C}$. The details have been published previously $^{19}$.

The low-dose and high-dose BNT162b1 vaccines contained $10 \mu \mathrm{g}$ and $30 \mu \mathrm{g}$ active ingredient, respectively, and the placebo was a commercial, pre-packaged preservative-free saline solution containing $0.9 \% \mathrm{NaCl}$. Each participant received a prime dose and a boost dose of vaccine candidate BNT162b1 at either $10 \mu \mathrm{g}$ in $0.5 \mathrm{ml}$ or $30 \mu \mathrm{g}$ in $0.5 \mathrm{ml}$, or $0.5 \mathrm{ml}$ placebo, administered into the deltoid, $21 \mathrm{~d}$ apart.

Monitoring of safety. Each participant was asked to remain at the study site for at least $6 \mathrm{~h}$ after vaccine administration for safety observation. Vital signs including temperature, blood pressure, pulse and respiratory rate were measured at baseline, $1 \mathrm{~h}, 3 \mathrm{~h}$ and $6 \mathrm{~h}$ after vaccination. Any adverse events following the vaccination were documented by participants using diaries until $28 \mathrm{~d}$ after the boost dose, in line with vaccine clinical development guidance and GCP inspection requirements in China. The participants in the younger age group were enrolled and received the vaccination first. Enrollment of the participants in the older age group was launched following evaluation of the preliminary safety data for the younger age group for the first $14 \mathrm{~d}$ after the prime vaccination. Severity of adverse events and laboratory abnormal changes are graded using a scale issued by the China State Food and Drug Administration ${ }^{24}$ and a scale issued by the US FDA ${ }^{23}$. Blood samples for safety laboratory testing were taken at baseline, $24 \mathrm{~h}$ after the prime dose, $\mathrm{d} 8$ and 29 , and at 6 months after the prime dose (thyroid function only). When necessary or when requested, adverse events were handled with simple standard of care.

All of the reported adverse events were reviewed by investigators. Adverse events were categorized as either possibly, probably or definitely related to the vaccine candidate.

A Safety Review Committee reviewed (blinded) safety and tolerability data following the prime vaccination and gave the go-ahead for the boost vaccination for each cohort.

Assessment of immunogenicity. Serum was collected at baseline and at d 8, 22, 29 and 43 to facilitate measurement of specific IgG antibody responses to RBD binding and spike glycoprotein $\mathrm{S} 1$ binding, and neutralizing antibody responses to
SARS-CoV-2. To assess T cell responses, $\mathrm{PBMC}$ samples were collected at baseline, d 29 (younger and older adults) and d 43 (younger adults only).

Human convalescent sera. A panel of 24 convalescent human serum samples was obtained from donors aged 18-70 years (mean age, 45.8 years) at least $14 \mathrm{~d}$ after a SARS-CoV-2 PCR-confirmed diagnosis of COVID-19. The disease severity of these patients varied from non-symptomatic $(n=3,13 \%)$ to mild $(n=8,33 \%)$, moderate $(n=10,42 \%)$ or severe $(n=3,13 \%)$

Neutralizing GMTs in subgroups of the donors were as follows: 40.0 for the three donors with non-symptomatic infections, 226.3 for the eight donors with mild infection, 91.9 for the ten donors with moderate infection, and 160.0 for the three donors with severe infection. Each serum sample in the panel was from a different donor. Thus, most of the serum samples were obtained from persons with moderate COVID-19. The convalescent serum samples were tested in microneutralization assay side by side as comparators with the serum samples obtained from participants in this trial.

ELISA. Total anti-SARS-CoV-2 antibodies were assessed using an indirect ELISA assay. In brief, serum samples were twofold serially diluted (1:100 to $1: 51,200)$ with sample diluent (Tris-buffered saline with 3\% BSA and 0.05\% Tween-20) and tested in 96 -well plates coated with recombinant RBD or S1 protein ( $100 \mathrm{ng}$ in $100 \mu \mathrm{l}$, Sino Biological) in sodium carbonate buffer. Bound IgG was detected using a horseradish peroxidase-conjugated secondary antibody (cat. no. 2049-05, Southern Biotech) diluted for each ELISA assay (anti-S1 detection, 1:40,000; anti-RBD detection, 1:20,000) and TMB substrate (Surmodics). Data collection was performed using a SpectraMax M5 reader (Molecular Device) of optical density (OD) at $450 \mathrm{~nm}$ using the SoftMax Pro GxP Software (version 6.5.1). To obtain a sample titer, the two points adjacent to the assay cutoff value were taken for linear fitting, and the sample dilution corresponding to the cutoff value was the titer value. The sample titer was set to 51,200 if its $\mathrm{OD}_{450}$ was greater than the cutoff value at 1:51,200 dilution; if the sample $\mathrm{OD}_{450}$ was less than the cutoff at an initial dilution of 1:100, an arbitrary titer of 50 (half of the limit of quantification) was reported.

Microneutralization assay. SARS-CoV-2-specific neutralizing antibody titer in serum was determined using a CPE-based microneutralization assay with the SARS-CoV-2 virus strain BetaCoV/Jiangsu/JS02/2020 (EPI_ISL_411952) and Vero-E6 cells (National Collection of Authenticated Cell Cultures, National Academy of Science, China). In brief, serum samples were heat-inactivated for $30 \mathrm{~min}$ at $56^{\circ} \mathrm{C}$ and twofold serially diluted from 1:10 to 1:5,120 using DMEM (Thermo Fisher Scientific). Serum dilutions were then mixed with the same volume of virus solution to achieve a $\operatorname{TCID}_{50}(50 \%$ tissue culture infectious dose) of 200 in each well. The serum-virus mixture was incubated at $37^{\circ} \mathrm{C}$ for $1 \mathrm{~h}$, then added to 96-well plates containing semi-confluent Vero-E6 cells ( $>80 \%$ density). After culturing at $37^{\circ} \mathrm{C}$ for $3 \mathrm{~d}$, CPEs on Vero-E6 cells were observed under an inverted microscope. The neutralizing titer is the reciprocal of the highest sample dilution that protects at least $50 \%$ of cells from CPE. If no neutralization reaction was observed at the initial serum dilution (1:10), an arbitrary titer of 5 (half of the limit of quantification) was reported.

IFN- $\boldsymbol{\gamma}$ ELISpot. SARS-CoV-2 S-specific T cell responses were assessed using an ex vivo IFN- $\gamma$ ELISpot assay (ELISpot ${ }^{\text {Pro }}$ Kit, cat. no. 3420-2AST-10, Mabtech). Tests were performed in triplicate with a positive control (anti-CD3 monoclonal antibody (mAb), diluted 1:1,000; mAb CD3-2, Mabtech). Per well, $1 \times 10^{5}$ freshly isolated PBMCs were stimulated for $21 \pm 0.5 \mathrm{~h}$ with one of three different peptide pools: two SARS-CoV-2 specific pools of 15 -mer peptides overlapping by 11 amino acids and together covering the whole sequence of wild-type SARS-CoV-2 S (Wuhan-Hu-1, GenBank QHD43416.1: Sp1 featuring amino acids 1-643, Sp2 featuring amino acids 633-1273) (PepMix SARS-CoV-2 (Spike Glycoprotein), PM-WCPV-S, JPT Peptide Technologies), or the CEF (CMV, EBV, influenza virus) HLA class I epitope peptide pool (CEF peptide pool for human CD8 T cells, 3616-1, Mabtech) as a positive control for general T cell reactivity. Bound IFN- $\gamma$ was visualized using a secondary antibody directly conjugated with alkaline phosphatase followed by incubation with 5-bromo-4-chloro- $3^{\prime}$-indolyl phosphate and nitro blue tetrazolium substrate (Mabtech, cat. no. 3420-2AST-10). Plates were scanned using an AID ELISPOT Reader (AID Autoimmun Diagnostika) and data were exported using AID EliSpot version 7.0. Spot counts were displayed as the mean of each triplicate, which was calculated by subtracting the mean negative control response from the mean of each peptide pool response.

Outcomes. The primary and secondary objectives of this trial were to evaluate the safety and immunogenicity of the candidate vaccine BNT162b1 in healthy Chinese adults. The primary endpoints for the safety evaluation were the incidence of solicited local reactions at the injection site or systemic adverse reactions in the $14 \mathrm{~d}$ after the prime or boost dose, and adverse events after the full immunization in the $28 \mathrm{~d}$ after receiving the boost dose. Any clinical laboratory abnormalities from baseline to $24 \mathrm{~h}$ or $7 \mathrm{~d}$ after the prime or boost dose, and any serious adverse event that occurred, were also recorded.

The secondary endpoints for immunogenicity were GMT, seroconversion rate and fold increase of virus-neutralizing antibody, or ELISA IgG antibodies 
binding to $\mathrm{S} 1$ or RBD measured $7 \mathrm{~d}$ and $21 \mathrm{~d}$ after both the prime dose and the boost dose. Seroconversion is defined as an increase by a factor of 4 or more in antibody titer over baseline, or the lower limit value if the baseline titer is below the limit of detection. The serum dilution for ELISA started at 1:100, while that for microneutralization assay started at 1:10.

Cellular immune responses in terms of the number of positive cells with IFN- $\gamma$ secretion in PBMCs at a concentration of $1 \times 10^{5}$ per well at $7 \mathrm{~d}$ after the boost dose (that is, $\mathrm{d} 29$ ), and for younger adults at $21 \mathrm{~d}$ after the boost dose (that is, $\mathrm{d} 43$ ), were investigated as an exploratory endpoint. The outcomes reported in this paper remained unaltered throughout the trial.

Statistical analysis. The total sample size in this study was 144 participants; that is, 24 participants of each age group were included in each of the three treatment groups. The probability to observe a particular adverse event with an incidence of $8 \%$ at least once in 24 participants in each dose group was $86.5 \%$.

All of the randomized participants who received at least one dose of the investigational vaccine were included in the safety analysis. Safety endpoints are described as frequency (\%) with the $95 \% \mathrm{CI}$ of the adverse reactions or events during the observation period. We compared the proportions of the participants with adverse reactions or events across the groups using the chi-squared test or Fisher's exact test. All of the participants who received at least one vaccination and who had results of serologic measurements at baseline or after vaccination were included in the immunogenicity analysis. The immunological endpoints were descriptively summarized at the specified time points. Comparisons of antibody titers between time points within the same group were performed using the Wilcoxon matched-pairs signed-rank test for non-normal data.

The peak neutralizing antibody responses of the participants in each dose group were compared with those of patients who had PCR-confirmed SARS-CoV-2 infection using the Mann-Whitney $U$-test. Analysis of non-normal ELISpot data was also performed using the Mann-Whitney $U$-test. Non-parametric Spearman correlation analysis of the 50\% neutralizing antibody GMTs and S1-binding antibody GMTs or T cell geometric mean IFN- $\gamma$ spot counts was performed to assess the relationship between responses in different assays.

Funding source. BioNTech RNA Pharmaceuticals and Shanghai Fosun Pharmaceutical Development funded this clinical trial. BioNTech was the regulatory sponsor, manufactured the BNT162b1 clinical trial material, advised on the study design, oversaw the trial execution, and contributed to the interpretation of the data and the writing of the manuscript. Shanghai Fosun was the execution organization of the trial. Fosun was responsible for the study design and conduct of the trial, data collection, data analysis, data interpretation and writing of the clinical study report, and advised on the manuscript. All of the authors had full access to all of the data in the trial and had final responsibility for the decision to submit the manuscript for publication.

Reporting Summary. Further information on research design is available in the Nature Research Reporting Summary linked to this article.

\section{Data availability}

We support data sharing of individual participant data. The individual participant data that underlie the results reported in this article (text, tables, figures and extended data) will be shared after de-identification. The raw data will be available for 1 year after the publication of this article. Researchers who provide a scientifically sound proposal will be allowed access to the individual participant data. Proposals should be directed to jszfc@vip.sina.com or aimin.hui@fosunpharma.com. Source data are provided with this paper.

\section{Code availability}

All code used to produce the results can be accessed by sending a scientifically sound proposal to jszfc@vip.sina.com or aimin.hui@fosunpharma.com. The code will be available with the raw data.

\section{Acknowledgements}

We thank M. Bushway, E. Derhovanessian and D. Maurus for critically reviewing the manuscript, M. Bexon for critically reviewing the manuscript and for his contribution to the planning and design of the study, and A. Finlayson for editorial and medical writing support. We also thank L. Zhu for providing the convalescent serum for the microneutralization assay. This trial was funded by Fosun and BioNTech and was registered under ChiCTR2000034825 at ChiCTR.

\section{Author contributions}

F.Z. is the principal investigator of this trial. J.L. worked as co-principal investigator of this trial. F.Z., A.H., J.L. and Y.Y. designed the trials and the study protocol. J.L. drafted the manuscript. A.H., L.Z., E.L. and A.M. contributed to the critical review and revision of the report. U.Ş. and Ö.T. designed and manufactured the vaccine candidate, and provided feedback on the study design. X.Z., R.T., H.Y., R.J., M.L., Z.Z., S.J., H.P., F.P., Z.M., Z.W. and Y.S. led and participated in the site work, including the recruitment, follow up and data collection. R.J., X.G. and L.Z. were responsible for laboratory analyses.

\section{Competing interests}

A.H., Y.Y. and R.J. are employees of Fosun Pharma and may hold stock options. U.Ş. and Ö.T. are stock owners, management board members and employees at BioNTech SE (Mainz, Germany), and are inventors on patents and patent applications related to RNA technology. E.L. and A.M. are employees at BioNTech SE (Mainz, Germany). All other authors have no competing interests.

\section{Additional information}

Extended data are available for this paper at https://doi.org/10.1038/ s41591-021-01330-9.

Supplementary information The online version contains supplementary material available at https://doi.org/10.1038/s41591-021-01330-9.

Correspondence and requests for materials should be addressed to A.H., L.Z. or F.Z.

Peer review information Nature Medicine thanks Elias Haddad, Nicole Frahm, Victor Volovici and the other, anonymous, reviewers for their contribution to the peer review of this work. Alison Farrell is the primary editor on this article and managed its editorial process and peer review in collaboration with the rest of the editorial team.

Reprints and permissions information is available at www.nature.com/reprints. 


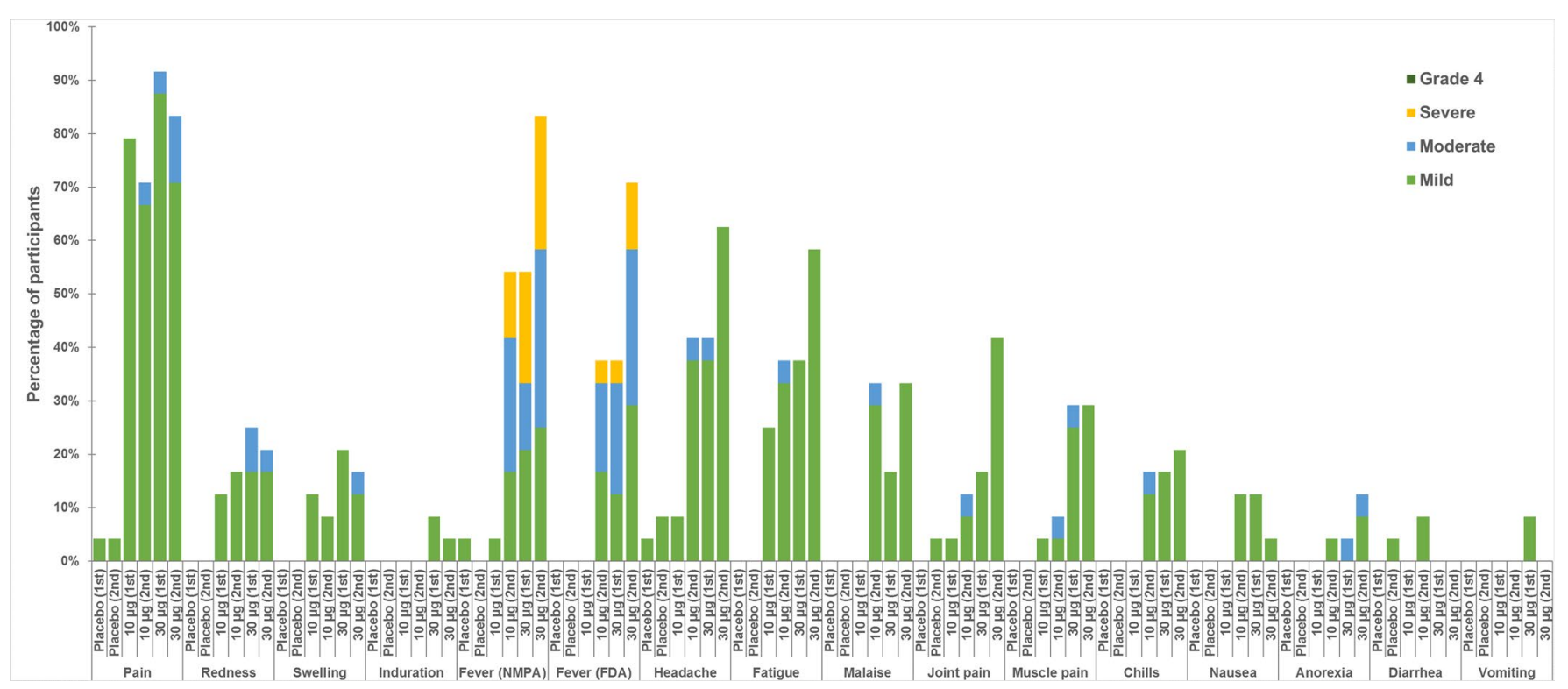

Extended Data Fig. 1 | Incidence of adverse reactions reported within 14 days of each vaccination with placebo, 10 or $30 \mu \mathrm{g}$ BNT162b1 in younger participants aged 18-55 years. Fever was graded according to both NMPA criteria and FDA criteria. Other adverse reactions were graded according to the grading guidelines for adverse events in vaccine clinical trials, issued by National Medical Products Administration (NMPA), China. 


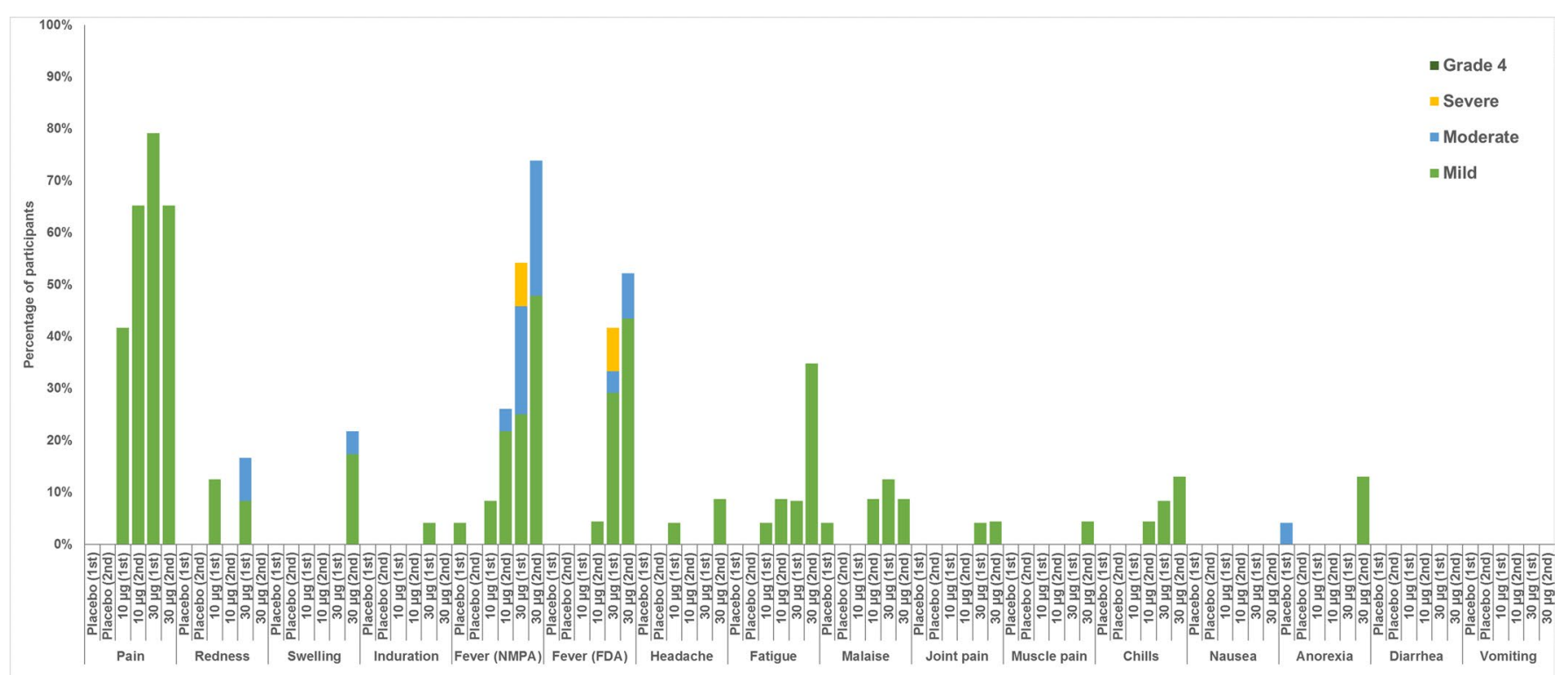

Extended Data Fig. 2 | Incidence of adverse reactions reported within 14 days of each vaccination with placebo, 10 or $30 \mu \mathrm{g}$ BNT162b1 in older participants aged 65-85 years. Fever was graded according to both NMPA criteria and FDA criteria. Other adverse reactions were graded according to the grading guidelines for adverse events in vaccine clinical trials, issued by National Medical Products Administration (NMPA), China. 
a Younger participants (18-55 years) Elderly participants (65-85 years)

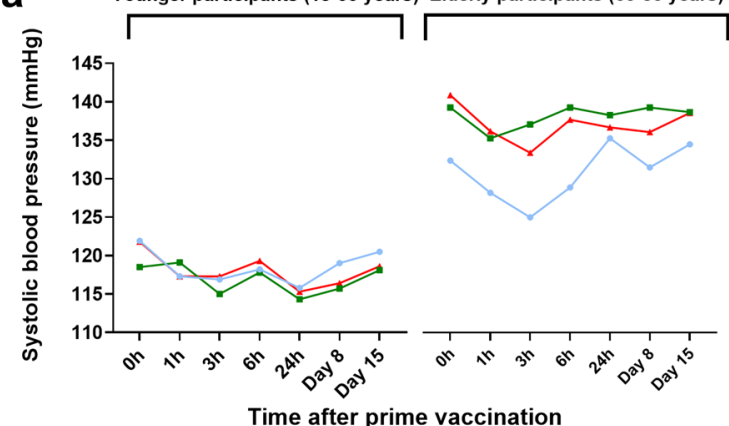

C

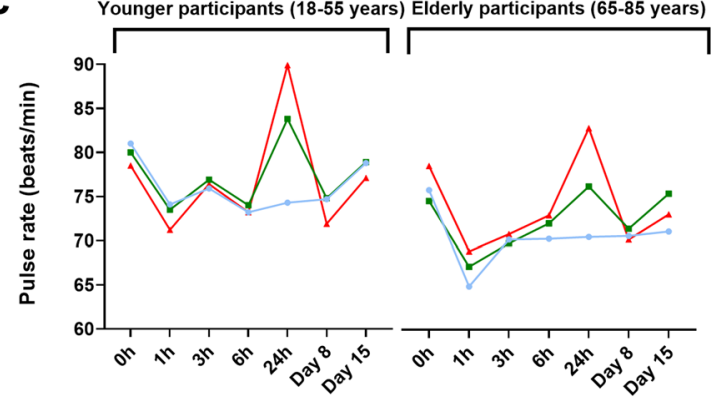

Time after prime vaccination

C Younger participants (18-55 years) Elderly participants (65-85 years)

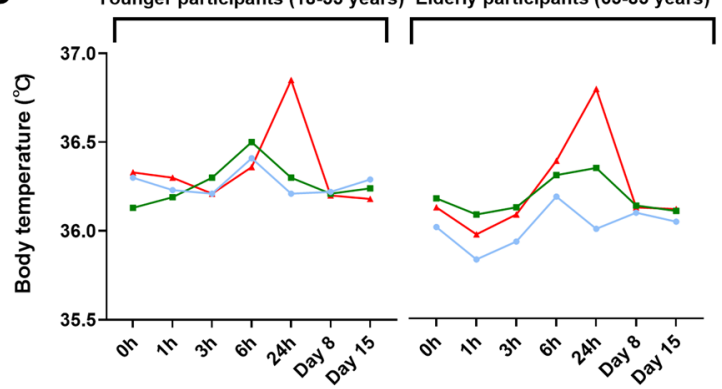

Time after prime vaccination b Younger participants (18-55 years) Elderly participants (65-85 years)

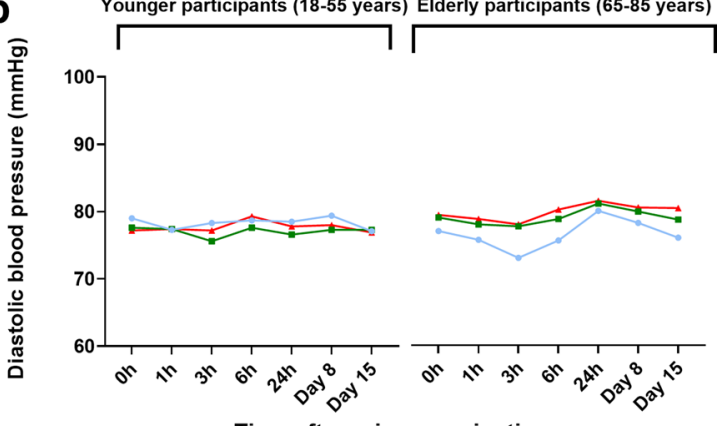

d

Time after prime vaccination

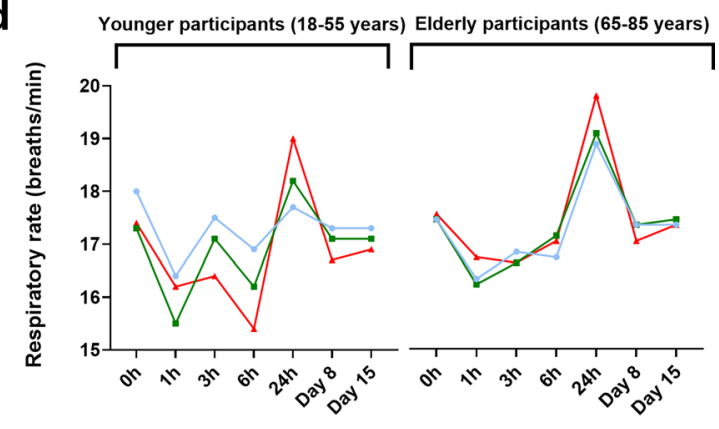

Time after prime vaccination

- Placebo

$-10 \mu \mathrm{g}$

$+30 \mu \mathrm{g}$

Extended Data Fig. 3 | Changes in vital signs measured in younger and older participants before and after prime vaccination. Vital signs including systolic blood pressure (a), diastolic blood pressure (b), pulse rate (c), respiratory rate (d), and body temperature (e) were tested before vaccination and at hours 1, 3, 6, 24, and days 7 and 14 after the prime dose of BNT162b1 at $10 \mu \mathrm{g}$ or $30 \mu \mathrm{g}$, or placebo. Data are shown as group mean. 
Younger participants (18-55 years) Elderly participants aged 65-85 years
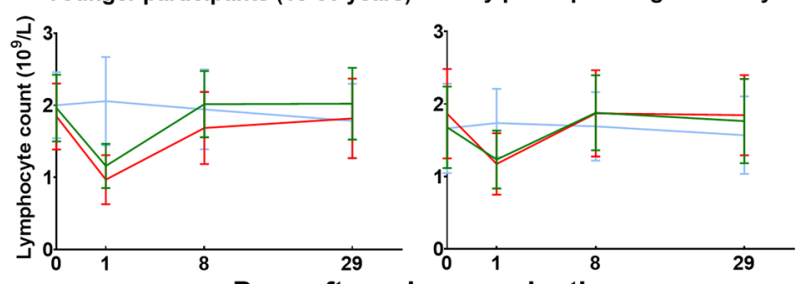

c

Younger participants (18-55 years) Elderly participants (65-85 years)
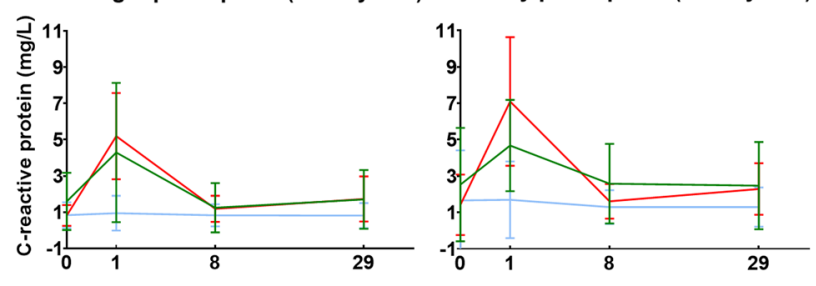

Days after prime vaccination

e

Younger participants (18-55 years) Elderly participants (65-85 years)

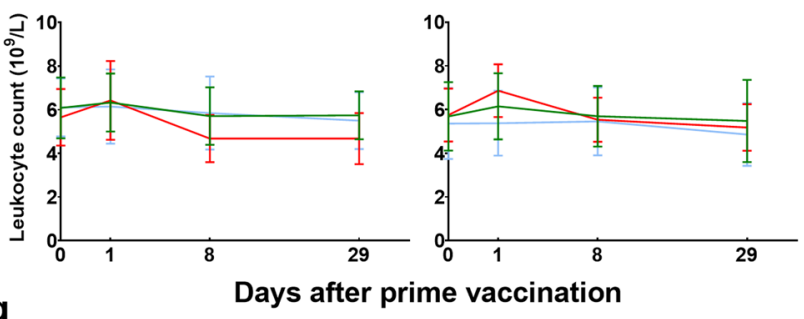

g

Younger participants (18-55 years) Elderly participants (65-85 years)

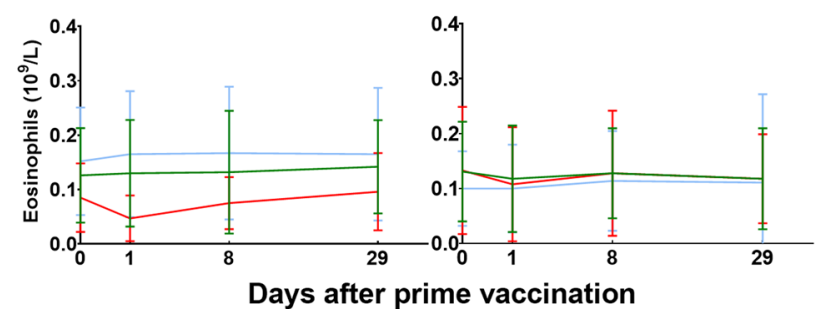

b

Younger participants (18-55 years) Elderly participants (65-85 years)

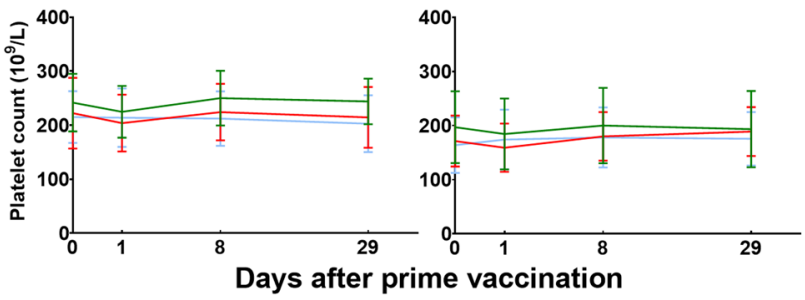

d

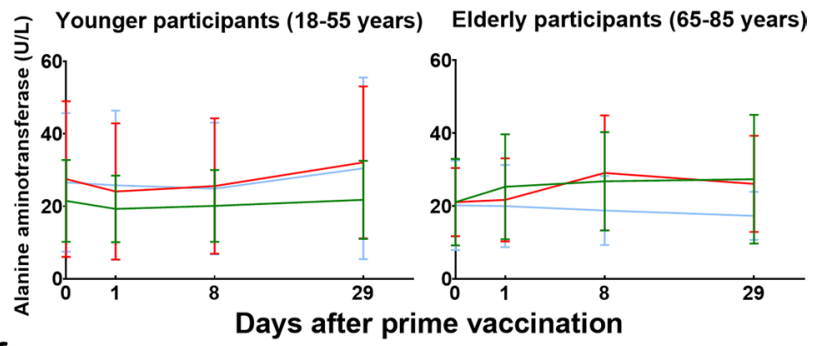

f

Younger participants (18-55 years) Elderly participants (65-85 years)
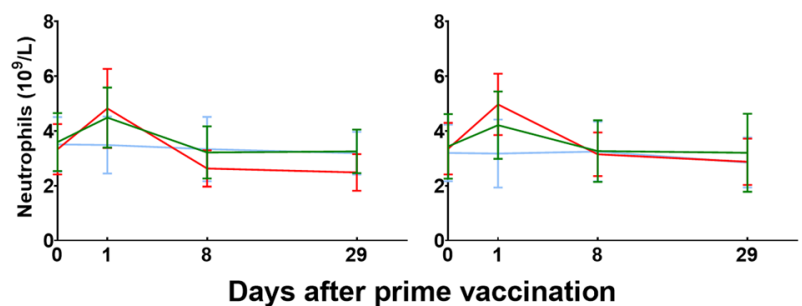

Placebo

$-10 \mu \mathrm{g}$

$-30 \mu \mathrm{g}$

Extended Data Fig. 4 | Changes of laboratory values from baseline measured in younger and older participants before and after prime vaccination. (a) Lymphocyte count, (b) platelet count, (c) C-reactive protein levels, (d) alanine aminotransferase levels, (e) leukocyte count, (f) neutrophil count, and (g) eosinophil count at day 0, and days 1, 8 and 29 after prime vaccination in younger and older participants. Data are shown as group mean with standard deviation. 
a Younger participants (18-55 years) VNT 50

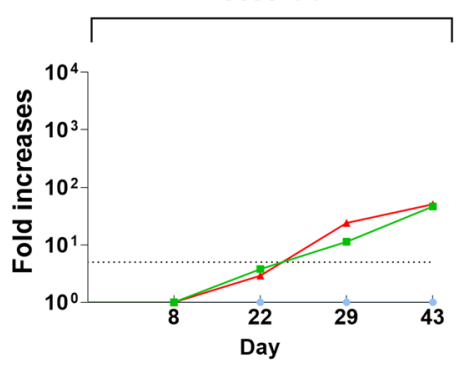

b Elderly participants (65-85 years)
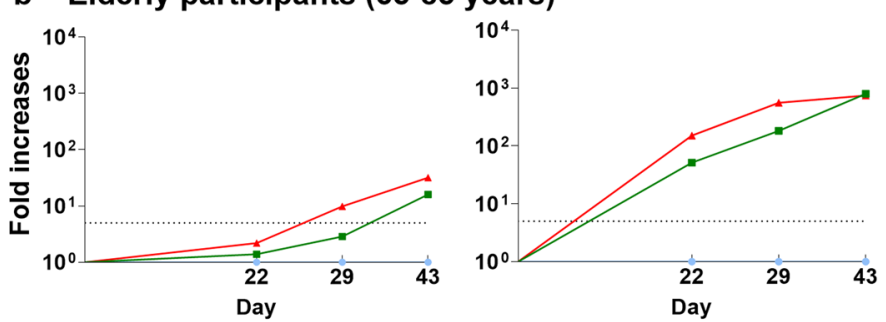

RBD Ab

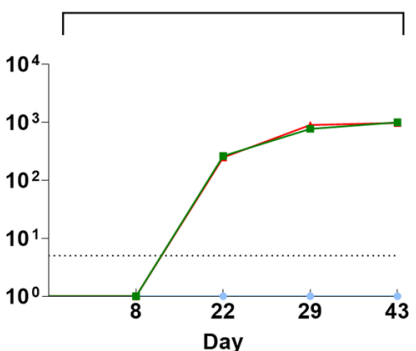

3
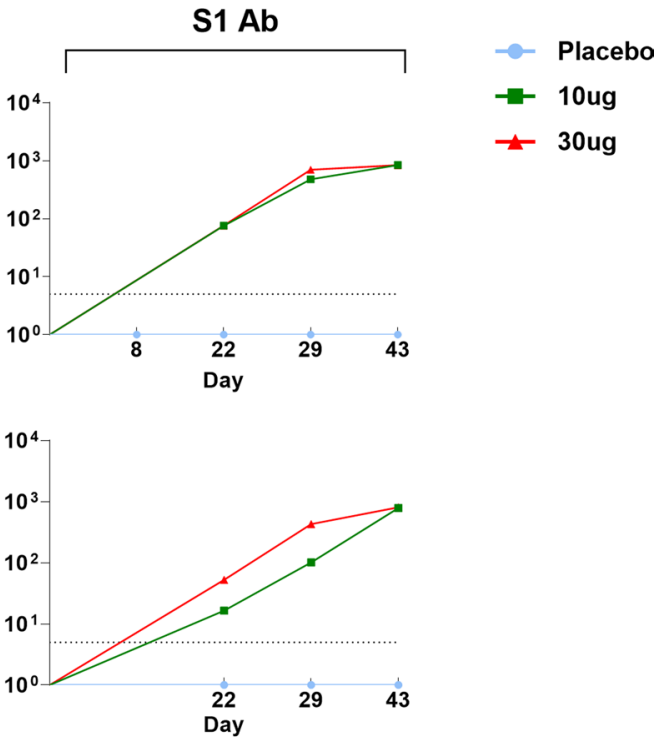

C Younger participants (18-55 years)
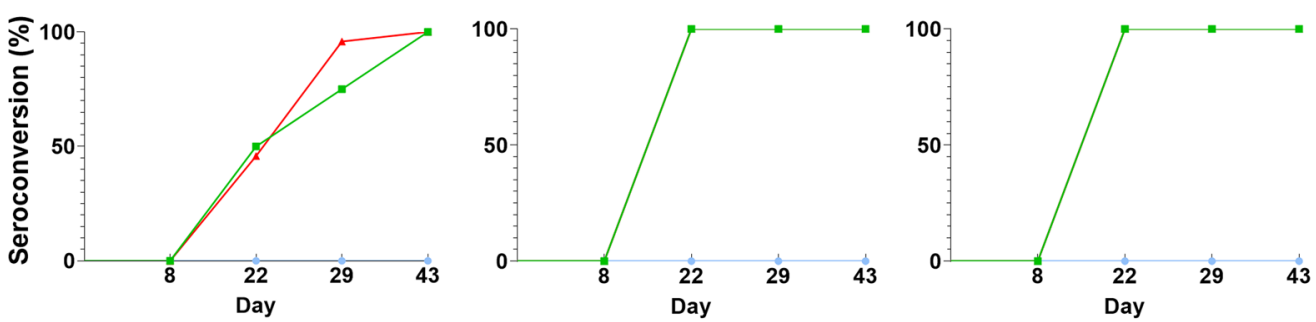

d Elderly participants (65-85 years)
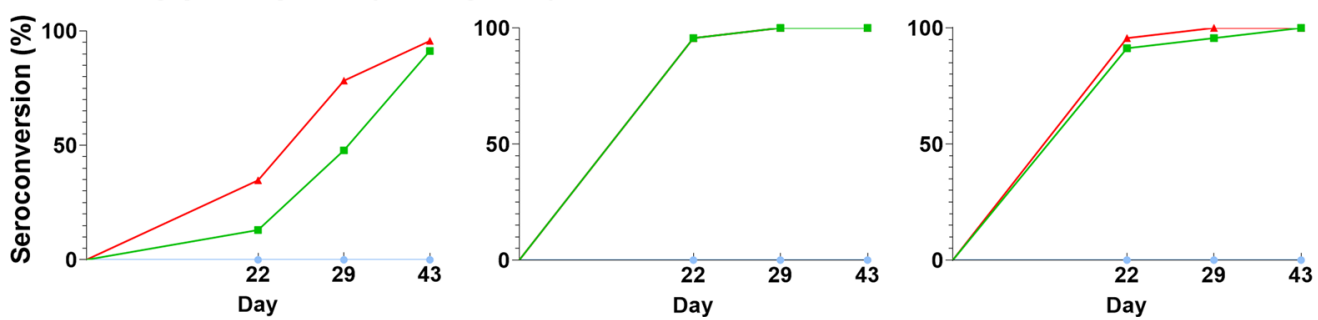

Extended Data Fig. 5 | Seroconversion and fold increases of specific neutralizing antibodies and binding antibody responses to RBD and S1 in participants. Serum samples were obtained at baseline (pre-prime, day 1), days 8 and 22 (pre-boost), and days 29 and 43 (post-boost) in the younger adult group, and at baseline (pre-prime, day 1), day 22 (pre-boost), and days 29 and 43 (post-boost) in the older adult group. Fold increase of neutralizing antibody and binding antibody responses in younger adult (a) and older adult participants (b). Fraction of seroconverted younger adult (c) and older adult participants (d) in per cent. Seroconversion was defined as at least a four-fold increase in post-vaccination titer from baseline or the lower limit of quantification, with a dashed line at the $\mathrm{Y}$-axis in (a) and (b) representing a four-fold increase in the specified antibody titer. Data are shown as group mean. VNT $50=50 \%$ virus neutralizing titer; RBD Ab $=$ Receptor binding domain-binding IgG titer; S1 Ab = S1-binding IgG titer. 
a Younger participants aged 18-55 years

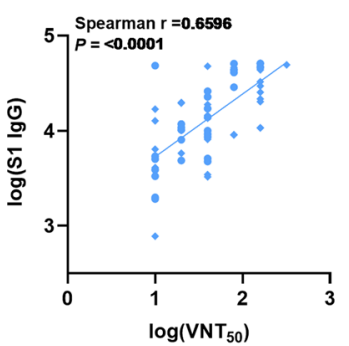

b Elderly participants aged 65-85 years

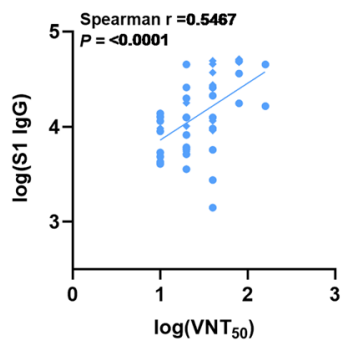

C Younger participants aged 18-55 years

\section{Day 29}

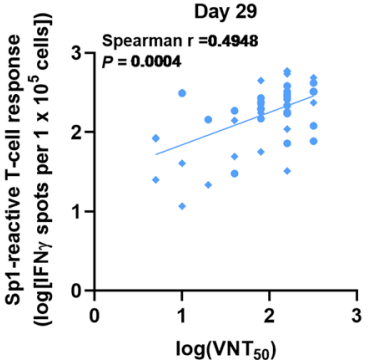

d Elderly participants aged $65-85$ years

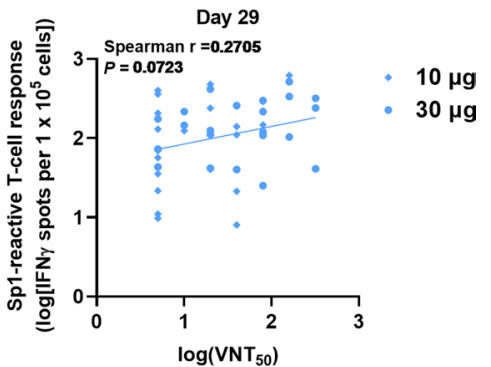

Extended Data Fig. 6 | Correlation of antibody and T-cell responses. a,b, Correlation of S1-specific lgG responses with 50\% SARS-CoV-2 neutralizing antibody responses in younger participants (a) and older participants (b). S1-specific IgG responses as in Fig. 2c, neutralizing antibody responses as in Fig. 2a. Data collected at Day 22 (pre-boost), Day 29 and Day 43 (7 and 21 days post-boost) were included, excluding those data sets with either one of the two values being non-evaluable. c,d, Correlation of S pool 1 (Sp1)-specific T cell responses with 50\% SARS-CoV-2 neutralizing antibody responses in younger participants (c) and older participants (d). S1-specific lgG responses as in Fig. 2c., Sp1-specific T cell responses as in Fig. 3a,b. Data collected at Day 29 (7 days post-boost) was included, excluding those data sets with either one of the two values being non-evaluable. Nonparametric Spearman correlations are shown. 


\section{Reporting Summary}

Nature Research wishes to improve the reproducibility of the work that we publish. This form provides structure for consistency and transparency in reporting. For further information on Nature Research policies, see our Editorial Policies and the Editorial Policy Checklist.

\section{Statistics}

For all statistical analyses, confirm that the following items are present in the figure legend, table legend, main text, or Methods section.

n/a Confirmed

$\bigotimes$ The exact sample size $(n)$ for each experimental group/condition, given as a discrete number and unit of measurement

$\square$ \ A statement on whether measurements were taken from distinct samples or whether the same sample was measured repeatedly

The statistical test(s) used AND whether they are one- or two-sided

Only common tests should be described solely by name; describe more complex techniques in the Methods section.

Х $\square$ A description of all covariates tested

Х $\square$ A description of any assumptions or corrections, such as tests of normality and adjustment for multiple comparisons

$\square$ A full description of the statistical parameters including central tendency (e.g. means) or other basic estimates (e.g. regression coefficient)

AND variation (e.g. standard deviation) or associated estimates of uncertainty (e.g. confidence intervals)

For null hypothesis testing, the test statistic (e.g. $F, t, r$ ) with confidence intervals, effect sizes, degrees of freedom and $P$ value noted Give $P$ values as exact values whenever suitable.

Х $\square$ For Bayesian analysis, information on the choice of priors and Markov chain Monte Carlo settings

$\square$ \ $\square$ or hierarchical and complex designs, identification of the appropriate level for tests and full reporting of outcomes

$\square$ Estimates of effect sizes (e.g. Cohen's $d$, Pearson's $r$ ), indicating how they were calculated

Our web collection on statistics for biologists contains articles on many of the points above.

\section{Software and code}

Policy information about availability of computer code

Data collection Inform (for data collected in the case report form) and electronic diary (Signant Health platform) for participant self reported reactogenicity Data collection for ELISA was performed using a SpectraMax M5 reader (Molecular Device, USA) at OD $450 \mathrm{~nm}$ using the SoftMax Pro GXP Software.

Data collection for the IFNgamma ELIspot assay were performed an AID ELISPOT Reader (AID Autoimmun Diagnostika, Germany) and data exported using AID EliSpot software Version 7.0

No custom software codes have been developed.

Data analysis

SAS 9.4 was used to manage, analyze and export patient-level clinical trial data.

All statistical analyses were performed using GraphPad Prism software version 8.4.2.

For manuscripts utilizing custom algorithms or software that are central to the research but not yet described in published literature, software must be made available to editors and

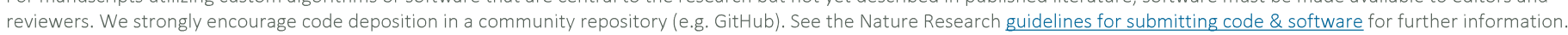

Data

Policy information about availability of data

All manuscripts must include a data availability statement. This statement should provide the following information, where applicable:

- Accession codes, unique identifiers, or web links for publicly available datasets

- A list of figures that have associated raw data

- A description of any restrictions on data availability

We support data sharing of individual participant data. The individual participant data that underlie the results reported in this article, after de-identification (text, tables, figures, and extended data) will be shared. The raw data will be available after the publish of this article till one year after publication. Researchers who 


\section{Field-specific reporting}

Please select the one below that is the best fit for your research. If you are not sure, read the appropriate sections before making your selection.

$\bigotimes$ Life sciences $\quad \square$ Behavioural \& social sciences $\square$ Ecological, evolutionary \& environmental sciences

For a reference copy of the document with all sections, see nature.com/documents/nr-reporting-summary-flat.pdf

\section{Life sciences study design}

All studies must disclose on these points even when the disclosure is negative.

Sample size The total sample size in this study was 144 participants, 24 participants of each age group was included in each treatment group. The probability to observe a particular adverse event with incidence of $8 \%$ at least once in 24 participants in each dose group was $86.5 \%$.

Data exclusions All safety and immunogenicity data were included in the report. No data were excluded from the analyses.

Replication This is an interim report of an ongoing human clinical trial. There was no attempt at replication of study findings.

Randomization This is an randomized controlled trial. We randomly assigned participants in each age group in a ratio of 1:1:1 to receive the low-dose BNT126b1 or high-dose BNT126b1 or placebo, stratified by gender, using a Web-based interactive response technology (IRT) system.

Blinding Authorized unblinded pharmacists prepared the vaccines or placebo according to the allocation of participants through the IRT system, and nurses administrated the investigational products to participants. The unblinded staff had no further involvement in the trial, and were forbidden to disclosure allocation information to others. All other investigators, participants, laboratory staff and the sponsor remained blinded throughout the trial.

\section{Reporting for specific materials, systems and methods}

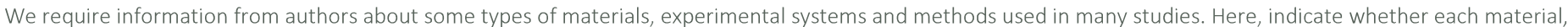

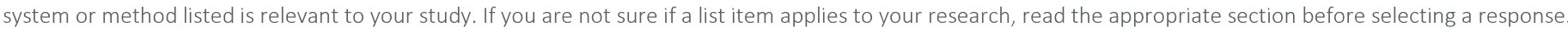

\begin{tabular}{l|l|l} 
Materials \& experimental syste \\
\hline$n / a$ & Involved in the study \\
\hline & $\square$ Antibodies \\
\hline & $\square$ Eukaryotic cell lines \\
$\square$ & $\square$ Palaeontology and archaeology \\
$\square$ & $\square$ Clinical data \\
$\square$ & $\square$ Dual use research of concern
\end{tabular}

\begin{tabular}{l|l} 
Methods \\
\hline n/a & Involved in the study \\
\hline & $\square$ ChIP-seq \\
$\square$ & $\square$ Flow cytometry \\
\end{tabular}

Eukaryotic cell lines

Policy information about cell lines

Cell line source(s)

Vero-E6 cells (National Collection of Authenticated Cell Cultures, National Academy of Science, China)

Authentication

The cell line was not authenticated

Mycoplasma contamination

The cell line was not tested

Commonly misidentified lines (See $\underline{I C L A C}$ register)

Name any commonly misidentified cell lines used in the study and provide a rationale for their use.

\section{Human research participants}

Policy information about studies involving human research participants

Population characteristics

144 eligible participants consented to participate in the trial and were randomized 1:1:1 to receive prime and boost doses of BNT162b1 at $10 \mu \mathrm{g}$ or $30 \mu \mathrm{g}$, or two placebo doses 21 days apart (Figure 1), with an equal allocation for each age group. 
Recruitment

Following priming doses, two participants (one at $10 \mu \mathrm{g}$, one at $30 \mu \mathrm{g}$ ) between the ages of 65 and 85 years had withdrawn from boost dose administration

Participants were generally in good health as established by medical history, physical examination, and laboratory tests at the screening visit. Both male and female were included and agreed to have contraception during the trial. We excluded those who pregnant or breast-feeding or known infection with SARS-CoV-2. The eligibility of participants was confirmed by negative results from a commercial rapid diagnostic kit for IgM/IgG antibody to SARS-CoV-2 manufactured by Livzon diagnostics inc., Zhuhai, China), and pharyngeal swabs nucleic acid diagnostic test (manufactured by Fosun pharma, Shianghai, China), and no imaging features of COVID-19 in chest CT scan. Participants had serious cardiovascular disease, or some major chronic illnesses were also excluded.

Ethics oversight

The study was done in accordance with the Declaration of Helsinki and Good Clinical Practice. The trial protocol was reviewed and approved by the National Medical Products Administration, China, and the institutional review board of the Jiangsu Provincial Center of Disease Control and Prevention.

Note that full information on the approval of the study protocol must also be provided in the manuscript.

\section{Clinical data}

Policy information about clinical studies

All manuscripts should comply with the ICMJE guidelines for publication of clinical research and a completed CONSORT checklist must be included with all submissions

Clinical trial registration This trial was registered with the Chinese Clinical Trial Registry (ChiCTR2000034825) and with clinicaltrials.gov (NCT04523571).

Study protocol

The protocol has been submitted.

Data collection

Each participant was asked to remain at the study site for at least six hours post vaccine administration for safety observation. Vital signs including temperature, blood pressure, pulse, and respiratory rate were measured at baseline, one hour, three hours and six hours post-vaccination. Any adverse events following the vaccination were documented by participants using diaries until Day 28 post-administration of the boost dose. Serum were collected at baseline, days 8 and 22 after each dose.

Outcomes

The primary and secondary objectives of this trial were to evaluate safety and immunogenicity of the candidate vaccine BNT162b1 in healthy Chinese adults. The primary endpoints for safety evaluation were the incidence of solicited local reactions at the injection site or systemic adverse reactions within 14 days post-vaccination, and adverse events following the full immunization until 28 days after receiving the boost dose. Any clinical laboratory abnormalities from baseline to 24 hours or 7 days after vaccination, and any serious adverse event (SAE) that occurred were also recorded.

The secondary endpoints for immunogenicity were geometric mean titer (GMT), seroconversion rates and fold increase of virusneutralizing antibody, or ELISA IgG antibodies binding to S1 or RBD measured at days 8, 22 after each vaccination. Seroconversion is defined as an increase by a factor of four or more in antibody titer over the baseline, or the lower limit value if the baseline titer is below the limit of detection. The serum dilution for ELISA started at 1:100, while that for microneutralization assay started at 1:10. Cellular immune responses in terms of the number of positive cells with interferon gamma (IFN- $\gamma$ ) secretion among PBMCs at a concentration of $1 \times 10^{\wedge} 5 /$ well at Day 8 and 22 after the boost dose were explored as an exploratory endpoint. 